\title{
Non-local transport nature revealed by the research in transient phenomena of toroidal plasma
}

\author{
Katsumi Ida ${ }^{1,2}$ (D
}

Received: 6 August 2021 / Accepted: 11 January 2022 / Published online: 27 January 2022

(C) The Author(s) 2022

\begin{abstract}
The non-local transport nature revealed by the research in transient phenomena of toroidal plasma is reviewed. The following non-local phenomena are described: core temperature rise in the cold pulse, hysteresis gradient-flux relation in the modulation ECH experiment, and see-saw phenomena at the internal transport barrier (ITB) formation. There are two mechanisms for the non-local transport which cause nonlocal phenomena. One is the radial propagation of gradient and turbulence. The other is a mediator of radial coupling of turbulence such as macro/mesoscale turbulence, MHD instability, and zonal flow. Non-local transport has a substantial impact on structure formations in a steady state. The turbulence spreading into the ITB region, magnetic island, and SOL are discussed.
\end{abstract}

Keywords Toroidal plasma $\cdot$ Non-local transport $\cdot$ Turbulence spreading

\section{Introduction}

The study of the relation between the radial flux and radial gradient of plasma density, flow velocity, and temperature in the magnetically confined toroidal plasma is crucial for predicting the plasma parameters (density and temperature) in a future device for nuclear fusion. This is because the smaller radial heat flux determined by the heating power and larger temperature gradient are desirable for a fusion reactor with high efficiency. The study to clarify the physics mechanism determining the gradient-flux relation is called transport study. There are two concepts of particle, momentum, and heat transport in the toroidal plasma. One is local transport, where the local gradient solely determines the local radial flux with a diffusion coefficient based on Fick's laws. Here, the diffusion coefficient depends on the various local

\section{Katsumi Ida}

ida.katsumi@nifs.ac.jp; idakatsumi@icloud.com

1 National Institute for Fusion Science, Toki, Gifu 509-5292, Japan

2 The Graduate University for Advanced Studies, Toki, Gifu 509-5292, Japan 
plasma parameters and satisfying the local closure. A significant contribution of the non-diffusive term (radial flux which does not depend on the gradient) exists in particle, momentum transports. The other is non-local transport, where the local radial flux is determined globally (violation of local closure).

In general, it is not easy to identify the non-local transport in the steady state because the diffusion coefficient varies in radius, and any amount of radial flux can be reproduced by arbitrary values of the diffusion coefficient. Therefore, the nonlocal transport was identified by the research in transient phenomena. The transient phenomena which cannot be explained by the local transport are called non-local phenomena. The non-local transport and non-local phenomena are not identical. Non-local transport, which does not exhibit non-local phenomena, also exists.

Non-local phenomena were observed as the transient temperature rise in the plasma core triggered by the cooling at the plasma edge (Gentle et al. 1995). Since then, many non-local phenomena have been observed in various toroidal plasmas. The typical examples are (1) non-local core temperature rise, (2) the hysteresis of gradient-flux relation, and (3) see-saw phenomena. Although there are many experimental results on non-local phenomena, the research for non-local transport mechanisms is limited despite its importance. The understanding of non-locality phenomena and non-local transport is an emerging issue in magnetically confined toroidal plasmas (Ida et al. 2015a). There are two models for non-local transport mechanisms. One is the radial propagation of gradient and turbulence intensity. And the other is the mediator, which causes the radial turbulence coupling. The candidate for the mediators are, macro/mesoscale turbulence, MHD, and zonal flow.

Non-local transport has a significant impact on the structure formation in the steady-state. The impact appears as turbulence spreading at the boundary layer of transport such as the ITB foot and H-mode pedestal, and at the boundary of magnetic topology such as the magnetic island boundary and last closed flux surface. The violation of local closure and the non-local response observed in experiments and theoretical models on the mechanism for the non-local transport are described in the previous work (Ida et al. 2015a). This review paper describes the pioneering work on transient phenomena and the recent experimental results to study the mechanism for non-local transport. The impact of non-local transport on the structure formation of the internal transport barrier (ITB) and staircase are also discussed. Nonlocal transport nature (hysteresis gradient-flux relation) and non-local phenomena are described in sections II and III. The mechanism and impact of non-local transport are discussed in sections IV and V.

\section{Non-local transport nature: hysteresis gradient-flux relation}

\subsection{Definition of non-local transport}

Two processes cause the radial flux of density, momentum, and heat in the plasma in magnetically confined toroidal plasmas. One is a radial flux enhanced by the collisional process of ion and electron, called neoclassical transport. The other is a radial flux due to the turbulence of potential, density, and temperature in the plasma, and this is called a 
turbulent transport or anomalous transport. Figure 1 shows the elements that determine turbulent transport, and the difference between local transport and non-local transport is illustrated. Here, $\Gamma, P_{\phi}$, and $Q$ are the particle, momentum and energy (heat), respectively, and $n$ is electron/ion/impurity density $\left(n_{e}, n_{i}, n_{I}\right), v_{\phi}$ is the toroidal flow velocity, and $T$ is the electron/ion temperature $\left(T_{e}, T_{i}\right)$, respectively. In the turbulent transport, the amplitude and phase of the turbulence of potential, density, flow velocity, and electron/ ion temperature $\left(\delta \Phi, \delta n_{e}, \delta V_{\phi}, \delta T_{e}\right.$, and $\left.\delta T_{i}\right)$ are mainly determined by the gradients of density, flow velocity, and electron/ion temperature $\left(\nabla n_{e}, \nabla V_{\phi}, \nabla T_{e}\right.$, and $\left.\nabla T_{i}\right)$. The gradient and turbulence determine the particle flux, toroidal momentum flux, and electron/ion heat fluxes $\left(\Gamma, P_{\phi}, Q_{e}\right.$, and $\left.Q_{i}\right)$. The radial fluxes are balanced to the volume integrated particle source (fueling), momentum source (torque input), and heat source (heating) in the steady-state. These sources are externally given in the confined plasma. Therefore, both gradient and turbulence are determined consistently by the magnitude of these sources, such as fueling, torque input, and heating power.

As seen in Fig. 1, gradient, turbulence, and radial flux, balanced to the source, make a loop. When there is no radial interaction, these quantities are determined locally by Fick's law, and the transport is called local transport. The particle flux, momentum flux, and heat flux are expressed by the so-called transport matrix as

$$
\left(\begin{array}{c}
\Gamma\left(\rho_{1}\right) \\
P_{\phi}\left(\rho_{1}\right) \\
Q\left(\rho_{1}\right)
\end{array}\right)=-M \nabla\left(\begin{array}{c}
n\left(\rho_{1}\right) \\
v_{\phi}\left(\rho_{1}\right) \\
T\left(\rho_{1}\right)
\end{array}\right) .
$$

local transport

(local closure by Fick's Law)

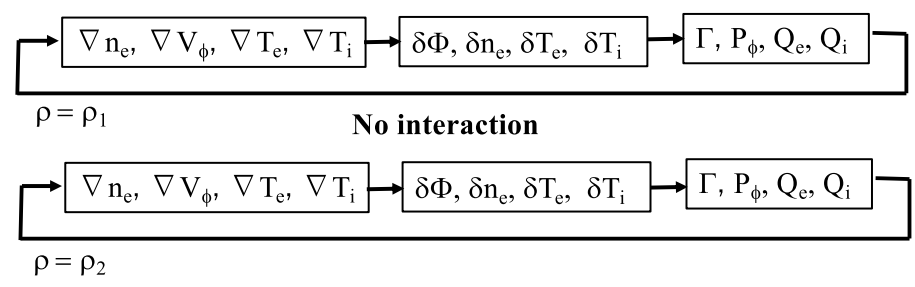

non-local transport (Violation of local closure by the breakdown of Fick's Law )

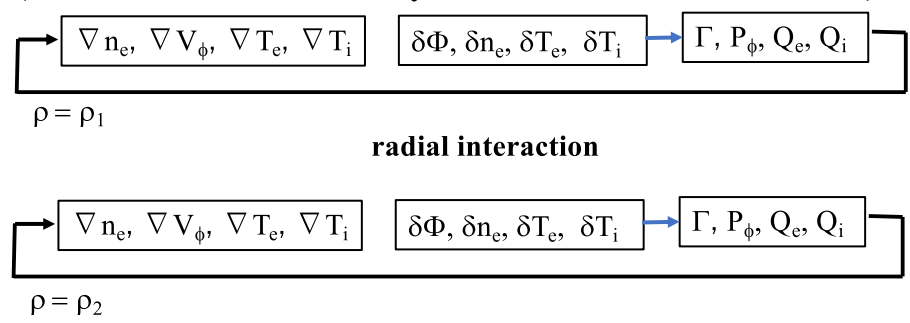

Fig. 1 Diagram of local and non-local transport 
The radial fluxes are determined uniquely by the gradients, and there is no hysteresis in the gradient-flux relation for the perturbation. That is $\Gamma\left(\rho_{1}\right), P_{\phi}\left(\rho_{1}\right), Q\left(\rho_{1}\right)$ are independent from $\nabla n_{e}\left(\rho_{2}\right), \nabla V_{\phi}\left(\rho_{2}\right), \nabla T\left(\rho_{2}\right)$.

In contrast, when there is radial interaction between two loops at different radii, Fick's law is the breakdown (Hahm and Diamond 2018). These quantities are not determined locally due to the violation of local closure, and the transport is called non-local transport. In the non-local transport, the magnitude of radial fluxes may have two values for the given gradient depending on the turbulence nearby, and hysteresis in the gradient-flux relation appears for the perturbation.

\subsection{Hysteresis of gradient-flux relation}

The non-local transport is one of the physical mechanisms of transport hysteresis in gradient-flux relation, as seen in Fig. 2. The gradient-flux relation of heat transport is determined by the so-called hidden parameters as well as gradients. Therefore, there are multiple gradient-flux relations (transport curves) depending on the value of hidden parameters $\left(p_{A}, p_{B}\right.$, and $\left.p_{C}\right)$. The gradient of off-diagonal terms (e.g., density gradient or flow velocity shear in the heat transport) are standard hidden parameters. The other well-known hidden parameters are magnetic shear and radial electric field that is not explicitly included in the element of the transport matrix. There are two types of transport curves, one is without offset (i.e., zero y-interception), as seen in Fig. 2, and the other is with finite offset (i.e., non-zero y-interception). The y-intercept of the transport curve corresponds to the non-diffusive term of transport. Although the non-diffusive term of heat transport is small, the non-diffusive term of momentum and particle transport is large enough to provide a significant radial flux even for zero gradient. For example, the radial momentum flux driven by intrinsic torque and radial particle flux driven by convection is the well-known

\section{Local transport}

local closure by Fick's Law

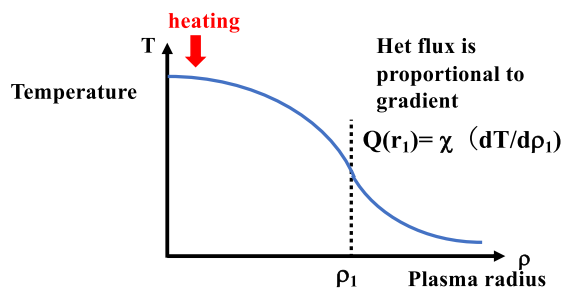

Hear flux

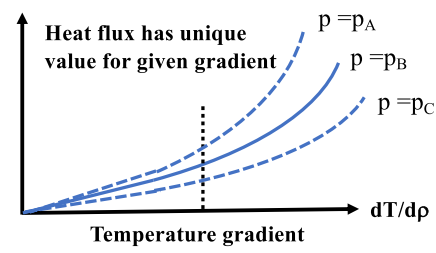

Non-local transport

Violation of local closure by the breakdown of Fick's Law
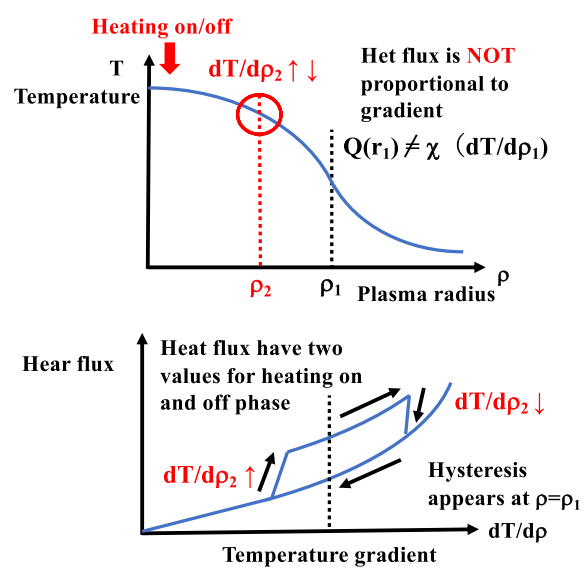

Fig. 2 Physics mechanism of hysteresis in gradient-flux relation 
non-diffusive term of transport. The bifurcation of transport reveals itself as the transition between two transport curves governed by the hidden variables. In heat transport, the bifurcation is observed as the jump of the gradient. The bifurcation of particle and momentum transports appears as a sign-flip of y-interception. When the forward and backward transitions repeat, the gradient-flux relation also shows the hysteresis characteristics. The most typical example of this hysteresis characteristic is limit cycle oscillation due to the jump of a radial electric field in the plasma with the heating power near the transition threshold.

The difference of hysteresis characteristics between local transport and non-local transport is whether the hidden parameter is a local parameter or not. In the case of hysteresis of local transport, the hidden parameters are local plasma parameters at the location the same as the gradient of the $x$-axis of the transport curves. In contrast, the hidden parameters of non-local transport hysteresis are the plasma parameters at a location different from that of the $x$-axis of the transport curves. (e.g., edge temperature for the core heat transport). In the steady state, it is impossible to find the hidden parameter of non-local transport. Therefore, the transient phenomena, either by the perturbation or by the transition, are necessary to identify non-local transport hysteresis. The edge cooling by impurity injection and modulated core heating by electron cyclotron heating $(\mathrm{ECH})$ are typical examples of perturbation. The transition from low-confinement (L-mode) to improved mode (internal transport barrier mode) is utilized to study non-local transport hysteresis.

\section{Non-local phenomena}

The nature and mechanism of non-local transport are summarized in Fig. 3. The various observations have identified the existence of non-local transport. The core temperature rise is the most common phenomenon, which reveals the non-local transport by the hysteresis in gradient-flux relation. This phenomenon is characterized by the spontaneous increase of core electron temperature after the transient edge cooling propagates inward from the plasma edge as a cold pulse.

The edge cooling has been produced by pellet injection or supersonic molecular beam injection (SMBI) in various toroidal plasmas (Gentle et al. 1995; Mantica et al. 1999; Tamura et al. 2005, 2007, 2010; Rice et al. 2013; Rodriguez-Fernandez et al. 2018). The modulation ECH is also a common technique to measure the hysteresis in gradient-flux relation at the mid-radius where there is no heat deposition (Stroth et al. 1996; Gentle et al. 2006; Inagaki et al. 2013). The heat flux starts to increase before the increase of temperature gradient after the $\mathrm{ECH}$ is turned on. In contrast, the heat flux starts to decrease before the decrease of temperature gradient after the ECH is turned off. Then, the heat flux in the time period for the ECH on-phase becomes larger than that in the time period for the $\mathrm{ECH}$ off-phase for the given temperature gradient. The heat flux at mid-radius increases due to the increase of temperature gradient near the plasma center not due to the increase of temperature gradient at mid-radius. This hysteresis clearly shows a coupling of heat flux at the mid-radius and temperature gradient near the plasma center, which is clear evidence of non-local transport. The abrupt spontaneous increase of temperature 
Table of nature and mechanism of Non-local transport

\begin{tabular}{l|lll}
\hline Transient transport experiment & & \\
\hline Technique & $\begin{array}{l}\text { Cold pulse by } \\
\text { pellet or SMBI }\end{array}$ & $\begin{array}{l}\text { Modulation } \\
\text { ECH }\end{array}$ & $\begin{array}{l}\text { Transport barrier } \\
\text { formation }\end{array}$ \\
\hline Observation & Core temperature rise & $\begin{array}{l}\text { Hysteresis of } \\
\text { gradient-flux relation }\end{array}$ & See-saw transport \\
\hline Propagation & Avalanche & Turbulence spreading & Gradient propagation \\
\hline Mediator & Zonal flow & $\begin{array}{l}\text { Macro/meso } \\
\text { turbulence }\end{array}$ & MHD instability \\
\hline Impact of Nonlocal transport & $\begin{array}{l}\text { Transport barrier } \\
\text { formation }\end{array}$ & $\begin{array}{l}\text { Turbulence in } \\
\text { magnetic island }\end{array}$ & Turbulence in \\
\hline Structure formation & & & SOL \\
\hline
\end{tabular}

Fig. 3 Table of nature and mechanism of non-local transport

gradient at the mid-radius is called an internal transport barrier (ITB) formation. The non-local transport is also observed in the transient phase at the ITB formation. After the formation of ITB, the thermal diffusivity coefficient decreases inside the ITB region but increases outside the ITB region. This simultaneous decrease/ increase of the thermal diffusivity coefficient is called see-saw transport.

In the mechanism causing the non-local transport, there are various types of propagation and types of mediators of radial interaction of turbulence. Turbulence spreading is a nonlinear coupling of fluctuation energy that redistributes the turbulence intensity field away from the regions where it is exciting (Lin and Hahm 2004; Gurucan et al. 2005). Avalanche is a ballistic front propagation of turbulence and gradient in the radial direction due to a strong nonlinearity of the growth rate of the micro-scale turbulence. Since the turbulence and gradient can be radially coupled at a distance much larger than the turbulence correlation length, this is categorized as turbulence spreading. The other radial propagation is due to the gradient propagation through the transient change in radial flux, which is an approach to explain the non-local phenomenon by the local transport model. There are various candidates for the mediator causing the radial interaction of micro-scale turbulence. Zonal flow is mesoscale shear flows driven by nonlinear interactions through energy transfer from micro-scale drift waves. Because of the energy transfer to and from the microscale turbulence, zonal flow can be one of the candidates for the mediator of turbulence coupling between two locations in the mesoscale. Macro-scale or mesoscale turbulence can also be a candidate for the mediator through the energy transfer by nonlinear interactions. Macro-scale or mesoscale MHD instability is another candidate for the mediator because of the interaction between MHD and micro-scale turbulence (Choi et al. 2021). 
Although the non-local transport is identified only in the transient phase, it strongly impacts the structure formation in the steady state. Since the turbulence is strongly suppressed in the ITB region, this is considered to be a linearly stable region. Then the turbulence spreading into the linearly stable regions (ITB ones) becomes crucial to determine the sharpness of the boundary between L-mode and the ITB region, which is known as the ITB foot. Since the magnetic island and scrape-off layer (SOL) are also linearly stable regions, the turbulence spreading into the magnetic island and SOL is a crucial issue. The turbulence spreading into the magnetic island strongly impacts the transport bifurcation inside the magnetic island (Ida et al. 2015b; Hahm et al. 2021). The turbulence spreading into SOL contributes to the enhancement of turbulence in the diverter leg and reduces the peak heat load at the diverter plate.

\subsection{Core temperature rise by edge cooling}

A core temperature rise by edge cooling is a non-local phenomenon identified in the experiment. The small amount of solid carbon performed the edge cooling as perylene in the Texas Experimental Tokamak (TEXT) or polystyrene in the Large Helical Device (LHD) injected into the plasma edge. In the LHD, the Tracer-Encapsulated Solid Pellet (TESPEL) system has been developed for impurity transport study. The TESPEL consists of polystyrene - $\mathrm{CH}(\mathrm{C} 6 \mathrm{H} 5) \mathrm{CH} 2-$ as an outer shell and impurities as an inner core as a tracer. The TESPEL without tracer impurity was used for the non-local transport experiment.

Figure 4 shows the experimental results of the non-local transport experiment in tokamak and helical plasmas. The carbon injection causes a sharp drop of electron temperature near the plasma edge at $\rho=0.84$ in TEXT. The electron temperature starts to decrease in the region of the outer half of the minor plasma radius $(\rho>0.5)$ by the cold pulse propagation to the inner area (Fig. 4a). In contrast, the electron temperature increases in the region of the inner half of the minor plasma radius $(\rho<0.5)$. This increase is due to the confinement improvement (reducing heat transport) because the heating power is constant in time. The radial profiles of electron thermal diffusivity that reproduce the time evolution of electron temperature measured are also plotted in Fig. 4b. Just after the cooling of the edge $(t=0.2,1.0 \mathrm{~ms})$ the electron thermal diffusivity increases transiently near the plasma edge $(\rho>0.8)$ but decreases in the plasma core region $(0.3<\rho<0.7)$. The decrease of thermal diffusivity in the core region becomes more significant later $(t=2.0 \mathrm{~ms})$, and the reduction of electron heat transport remains longer (up to $8 \mathrm{~ms}$ ).

A similar phenomenon was observed in the LHD, where there is no toroidal plasma current as seen in Fig. 4c, d, The observation of non-local phenomena in both tokamak and helical plasma shows that a core temperature rise by edge cooling is a universal phenomenon in toroidal plasma, not attributed to the plasma current in the tokamak. The time scale of this transient phenomena in the LHD is much longer than that in the TEXT due to the longer confinement time (larger plasma minor radius) in LHD. The core temperature rise by cooling the edge and reduction of electron thermal diffusivity near the plasma center $(\rho=0.19)$ remains up to 


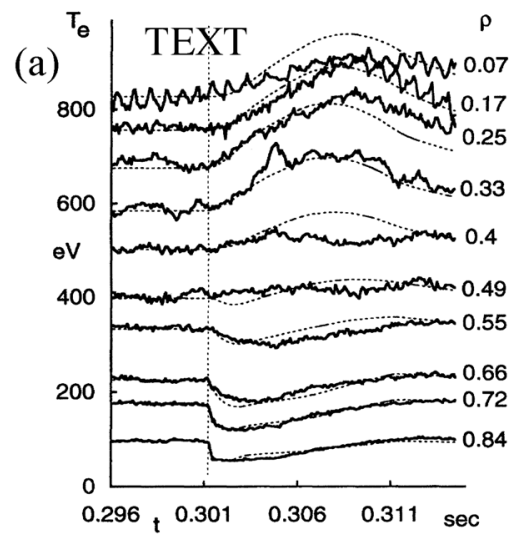

(c)

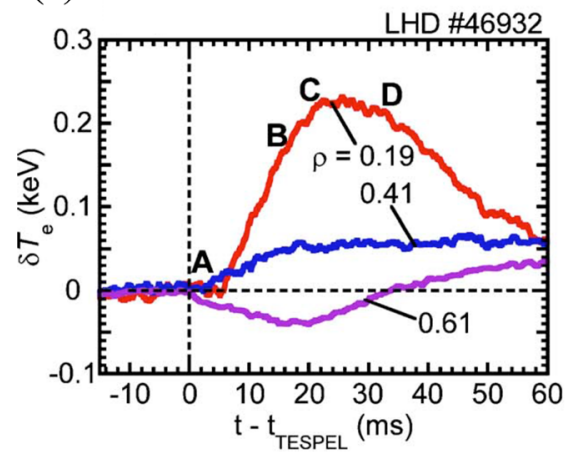

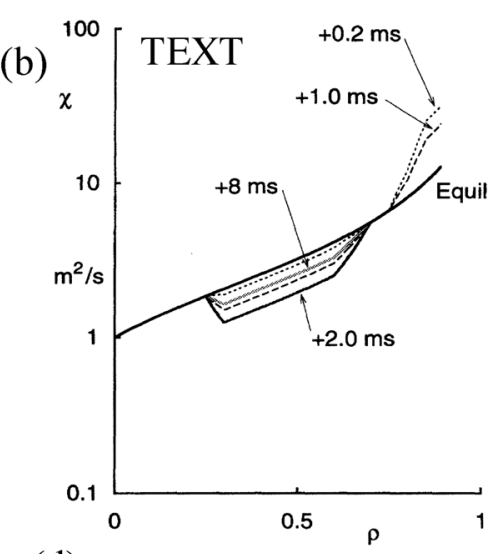

(d)

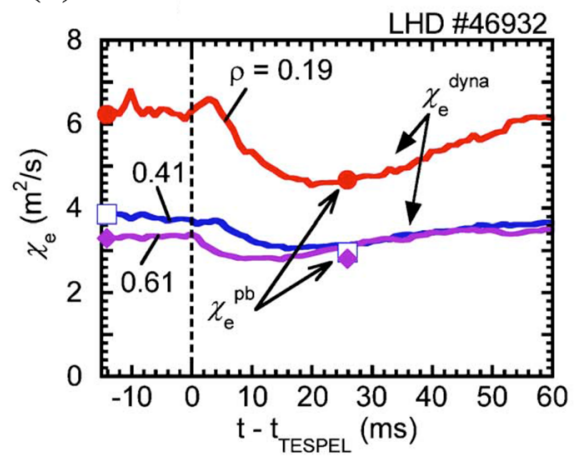

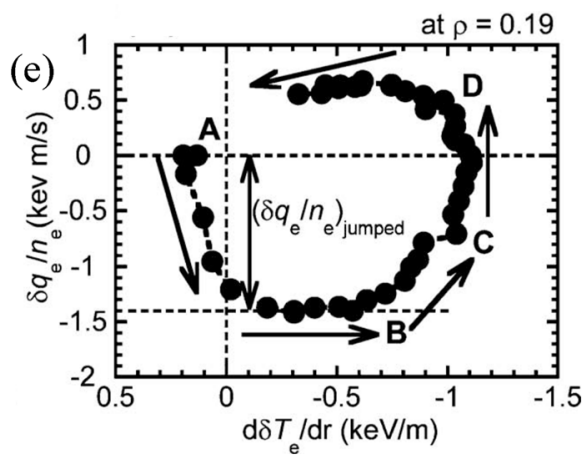

Fig. 4 a Time evolution electron temperature and $\mathbf{b}$ radial profile of electron thermal diffusivity in TEXT and time evolution of $\mathbf{c}$ the increment of electron temperature, (d) electron thermal diffusivity, and e gradient-flux relation of electron heat transport in LHD [from Figs. 2, 3 in (Gentle et al. 1995) and Fig. 2 in (Tamura et al. 2005)]

50-60 ms. Interestingly, the increase of electron temperature near the plasma center $(\rho=0.19)$ has a time delay of $5 \mathrm{~ms}$. The transient change in heat transport is more clearly observed in the gradient-flux relation as hysteresis rather than the reduction of heat diffusivity (the ratio of heat flux to the temperature gradient). As seen in the 
gradient-flux relation plotted (only transient changes of gradient and flux are plotted) in Fig. 4e, the decrease of thermal diffusivity in the earlier phase is due to heat flux reduction. In contrast, the further reduction of thermal diffusivity in the later stage up to timing B is due to the gradient increase. It should be noted that there are two stages even in the continuous decreasing phase of the thermal diffusivity phase. There are also two stages in the increasing phase (decay phase) of thermal diffusivity. The increase of thermal diffusivity is due to the increase of heat flux in the earlier stage and decreased temperature gradient in the later stage.

Various models are proposed to explain the non-local phenomena triggered by a cold pulse (del-Castillo-Negrete et al. 2005; Hariri et al. 2016; Fernandez et al. 2018). The trapped gyro-Landau fluid model, which contains a rule for turbulence saturation (TGLF-SAT1), was applied to model the cold pulse experiment in the Alcator C-mod laser blow-off experiment. In this experiment, density gradient propagates from the plasma edge to core after the laser blow-off. Figure 5 shows the time evolution of radial profiles of electron density and simulation results of electron and ion temperature during the density gradient propagation (Fernandez et al. 2018). Figure 5a shows that the density gradient peaked with a monotonic negative gradient before the density perturbation ( $t=0 \mathrm{~ms}$ ). After the Laser blow-off, the density
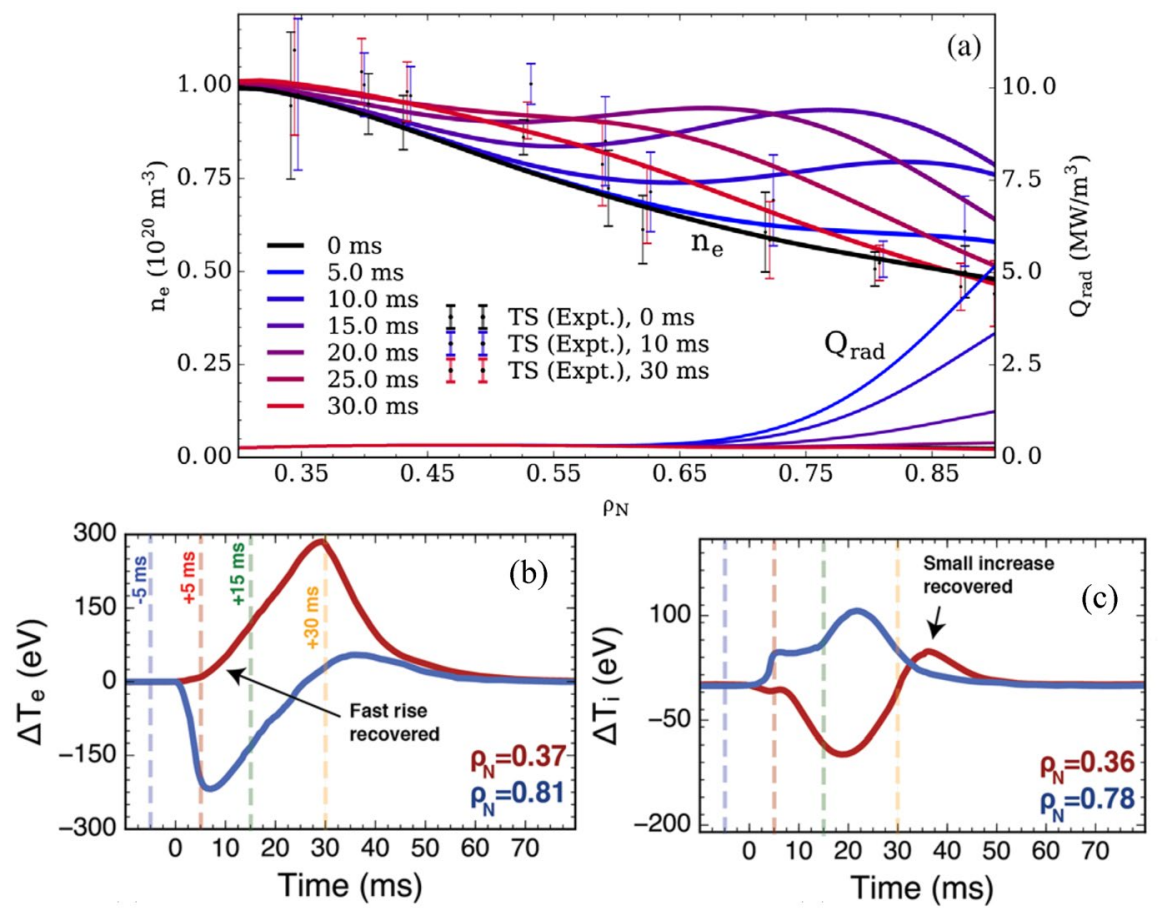

Fig. 5 a Radial profile of electron density and radiative sink, $\mathbf{b}$ time evolution of response of electron temperature at $\rho=0.37,0.81$ and $\mathbf{c}$ ion temperature at $\rho=0.36,0.78$ (from Figs. 2 and 4 in (Fernandez et al. 2018)) 
profile was significantly modified from peaked to hollow. The negative density gradient in the core $(\rho<0.5)$ becomes weak and density gradient in the outer region $(0.6<\rho<0.8)$ becomes positive transiently. The change in density gradient has a significant impact on the trapped electron mode (TEM) turbulence and ion temperature gradient (ITG) driven turbulence.

Figure $5 \mathrm{~b}, \mathrm{c}$ shows the time evolution of electron and ion temperature in the core ( $\rho=0.37$ and 0.36$)$ and near the edge $(\rho=0.81$ and 0.78$)$ simulated by TGLFSAT1 code. Interestingly, the change in density gradient has the opposite impact on electron and ion heat transport. The edge electron temperature drops sharply, and the core electron temperature gradually increases as the negative density gradient becomes weak in the later phase $(t=10-30 \mathrm{~ms})$. In contrast, the edge ion temperature increases rapidly and continues to increase up to $20 \mathrm{~ms}$. The core ion temperature decreases up to $30 \mathrm{~ms}$ and there is only a slight increase at the recovery phase. Rodriguez-Fernandez claims that the core electron temperature rises by edge cooling can be explained without a non-locality.

Non-local temperature rise in the ion heat transport has been reported both in tokamak and helical plasmas. Figure 6 shows the core ion temperature rise observed in the Alto Campo Toro C modification (Alcator C-mod) and the LHD. Cold pulses were achieved by the rapid edge cooling following $\mathrm{CaF} 2$ injection from a multipulse laser blow-off system in the Alcator C-mod and repetitive hydrogen pellet in the LHD. Non-local temperature rise appears in the low-density regime below the critical density for the transition from Linear ohmic confinement (LOC) to saturated ohmic confinement (SOC), as seen in Fig. 6a. Electron and ion temperatures increase in the core $(\rho=0.36$ and 0.1$)$ associated with the temperature drop near the plasma edge ( $\rho=0.86$ and 0.67 ). Because of the difference in the time delay of ion temperature rise of ion temperature and electron temperature, there is a period (
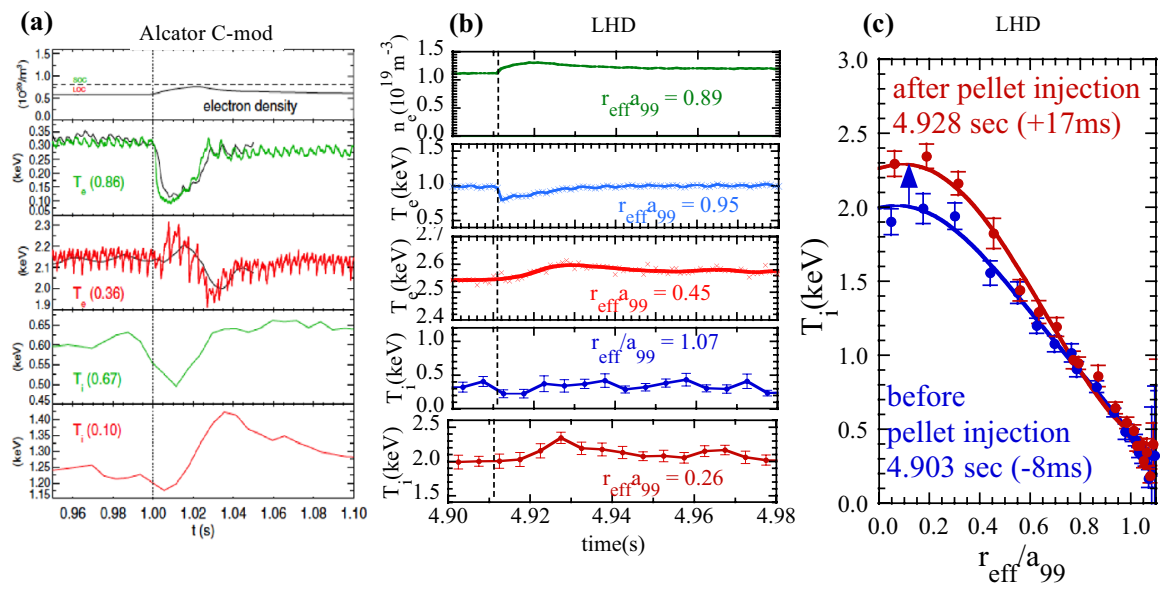

Fig. 6 a Time evolution of electron density, electron temperature at $\rho=0.86$ and 0.36 , ion temperature at $\rho=0.67$ and 0.10 in Alcator C-mod, and $\mathbf{b}$ time evolution of electron density, electron temperature at $\rho=0.95$ and 0.45 , ion temperature at $\rho=1.07$ and 0.26 , and (c) radial profile of ion temperature before and after the pellet injection in LHD [from figure 4 in (Rice et al. 2013)] 
$t=1.02-1.03 \mathrm{e} \mathrm{s}$ ) that ion temperature increases but electron temperature decreases in Alcator C-mod. This observation indicates that the radial coupling of the electron scale turbulence is different from that of the ion scale. The non-local ion temperature rise was also observed in the LHD as seen in Fig. 6b. Electron and ion temperatures increase in the core $(\rho=0.45$ and 0.26$)$ associated with the temperature drop near the plasma edge ( $\rho=0.95$ and 1.07). In the LHD, no difference in the time delay of ion temperature rise was observed. The region of ion temperature increase is a relatively wide region of the plasma minor radius $\left(r_{\text {eff }} / a_{99}<0.7\right)$ as seen in Fig. 6 (c) The transient rise of ion temperature in the core observed in the Alcator C-mod and LHD after the cold pulse contradicts the decrease of ion temperature in the core, simulated after the density perturbation in Fig. 5c.

\subsection{Hysteresis of gradient-flux relation induced by modulation ECH}

Modulation ECH has been commonly used in the non-local transport study because $\mathrm{ECH}$ provides a very localized perturbation in time and space. In this experiment, the heat flux is evaluated from the volume integrated heating power and time derivative of plasma energy. The deposition of the healing power of ECH can be highly localized near the plasma center. This is the great advantage in calculating the heat flux for ECH on-phase and off-phase precisely free from the uncertainty of the deposition profile at the mid-radius of the plasma.

Figure $7 \mathrm{a}$ shows the gradient flux relation of electron heat transport in the ECH modulation experiment in DIII-D. In this experiment, the modulation ECH was applied at $\rho=0.2$ with $0.7 \mathrm{MW}$ and the continuous ECH was applied at $\rho=0.3$ with $0.6 \mathrm{MW}$ in the plasma of averaged density of $2.5 \times 10^{19} \mathrm{~m}-3$. The hysteresis of gradient flux relation (multiple solutions of feat flux for the given gradient) and the jump pf heat flux at the switching of ECH is similar to that observed in Wendelstein 7 AS (Strorth et al., 1996). The jump of heat flux indicates that the difference between (1) the time derivative of plasma kinetic energy and (2) the increment volume integrated power inside the magnetic flux surface of the given minor radius is finite even if there is no change in gradient. In other words, the direct (not through conventional local transport) impact of heating appears even in regions where there is no deposition.

One candidate for the mechanism driving this direct impact is the thermodynamical force in plasma phase space controlling turbulence (Itoh and Itoh 2012). This model predicts the turbulence enhanced by the perturbation in phase space due to the heating and enhancement of turbulence not relating to the local gradient. Therefore, it is essential to study the behavior of turbulence in the ECH modulation experiment. The crucial question is whether turbulence modulation matches the local gradient phase at the given location. The turbulence behavior is investigated using a reflectometer outside the ECH deposition region in the LHD. Figure $7 b$ is the modified gradient flux relation, where nonlinearity of temperature and temperature gradient is taken into account with the power of $\alpha$ and $\beta$. The hysteresis remains for various sets of $\alpha$ and $\beta$. However, when the $x$-axis is replaced with the product of 

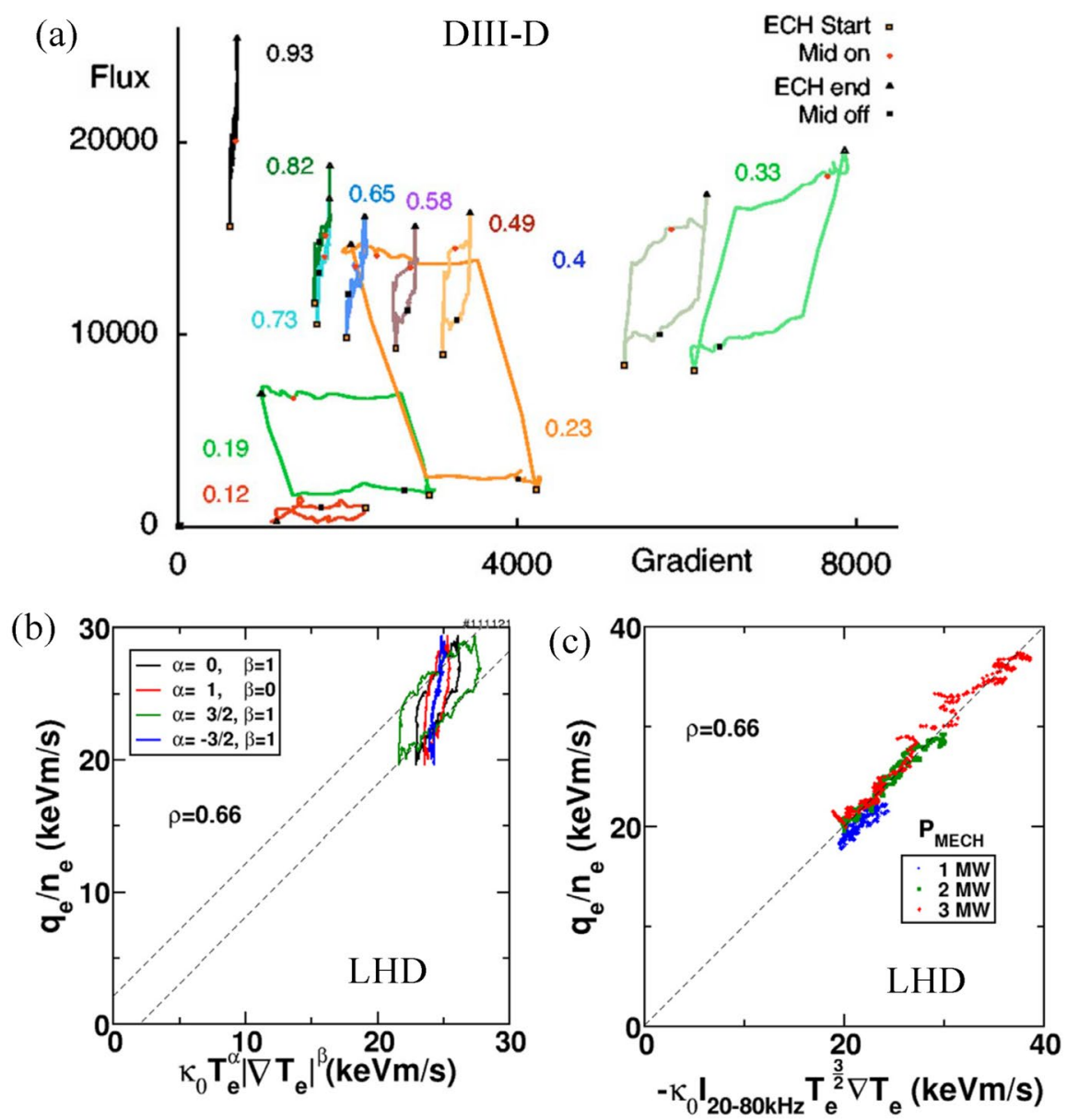

Fig. 7 a Gradient-flux relation of electron heat transport in DIII-D modulation ECH experiment and b, c gradient-flux relation of electron heat transport in LHD modulation ECH experiment (from Fig. 9 in [Gentle et al. 2006) and Figs. 8 and 11 in (Inagaki et al. 2013)]

turbulence intensity averaged over 20 to $80 \mathrm{kHz}$, temperature, and temperature gradient, the hysteresis disappears with the different levels of ECH power.

\subsection{See-saw transport}

See-saw transport is characterized by the simultaneous reduction and enhancement of transport at a different radius in the plasma. For example, the core confinement improvement and edge confinement degradation or vice versa is typical see-saw transport. Figure 8 shows the various examples of see-saw transport in tokamak and helical plasmas. See-saw transport phenomena are also observed after the cold pulse propagation, as seen in the radial profiles of electron temperature in Fig. 8a 

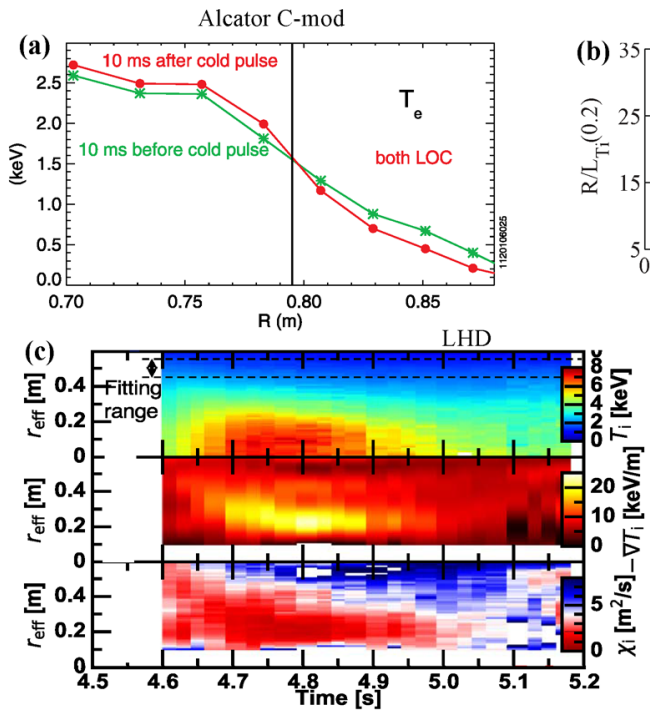

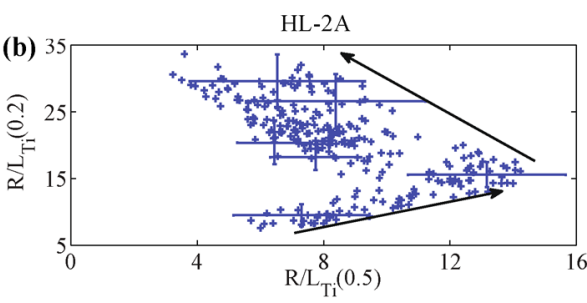

(d)

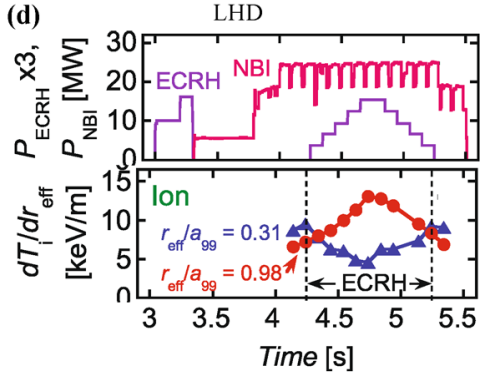

Fig. 8 a Radial profiles of electron temperature before and after could pulse in Alcator C-mod, b normalized ion temperature gradient at $\rho=0.2$ and 0.5 in HL-2A, c contour of ion temperature, ion temperature gradient, and ion thermal diffusivity, and $\mathbf{d}$ time evolution of ECRH and NBI power, ion temperature gradient at $r_{\text {eff }} / a_{99}=0.31$ and 0.98. (from Fig. 12 in (Rice et al. 2020), Fig. 8 in (Yu et al. 2016), Fig. 2 in (Kobayashi et al. 2019), and Fig. 4 in (Takahashi et al. 2017))

(Rice et al. 2020). The see-saw transport is often observed after the formation of the internal transport barrier (ITB) (Ida et al. 2009a, b; Yu et al. 2016; Kobayashi et al. 2019). Figure $8 \mathrm{~b}$ shows the relation between the normalized ion temperature gradient inside the ITG region $(\rho=0.2)$ and outside the ITB region $(\rho=0.5)$ in Huan-Liuqi-2A (HL-2A). The normalized ion temperature gradients increase both at $\rho=0.2$ and 0.5 before the formation of an ITB. The magnitude of normalized ion temperature gradients at $\rho=0.2$ is comparable to that at $\rho=0.5$. However, the simultaneous increase and decrease of normalized ion temperature gradients appear when the normalized ion temperature gradient at $\rho=0.2$ reaches a critical value of $\sim 15$. The normalized ion temperature gradients at $\rho=0.5$ starts to decrease, while the normalized ion temperature gradients at $\rho=0.2$ keeps increasing. Then the magnitude of normalized ion temperature gradients at $\rho=0.2$ becomes $\sim 7-8$ times larger than that at $\rho=0.5$, which clearly indicates the formation of ion ITB.

Enhancement of transport outside the ITB region during the formation of the ITB is also observed in the LHD, as seen in Fig. 8c. At the beginning of the ITB formation, the ion temperature profile becomes peaked, and the ion temperature gradient near the plasma core (at $\rho=0.2-0.3 \mathrm{e}$ ) increases. The core ion thermal diffusivity decreases but edge ion thermal diffusivity increases in time. In the LHD, the ITB is transient, and its region starts to shrink, and the region with enhanced transport expands from the plasma edge to mid-radius, and finally, the ITB disappears. The reduction of edge transport is observed in the plasma with ECH in the LHD, as seen in Fig. 8d In this experiment, the ion temperature gradient near the plasma 
center $\left(r_{\text {eff }} / a_{99}=0.31\right)$ decreases during the ECH power step up and increases during the ECH power step down. The change in ion temperature gradient is because the increase of $T_{e} / T_{i}$ by ECH heating causes the enhancement of ion transport as predicted by the characteristics of ITG turbulence. In contrast, the ion temperature gradient near the plasma edge $\left(r_{\text {eff }} / a_{99}=0.96\right)$ shows the behavior opposite to that near the plasma center. This experiment is an excellent example of see-saw transport for core confinement degradation and edge confinement improvement.

\section{Non-local transport}

\subsection{Radial propagation}

The fast propagation of gradient is the radial coupling (interaction) between two separated (longer than the correlation length) locations which should involve some process which is rigorously non-local. Figure 9 shows the non-local phenomena due to the radial propagation of gradients. A local gradient of density, toroidal flow velocity, and temperature determine the local particle, momentum, and heat flux in the steady state. However, in the transient phase, these radial fluxes alter the downward gradients. Therefore, the two loops at different radii can be coupled through the radial fluxes in the transient phase. Rigorously speaking, the coupling is not local transport because there is radial interaction of turbulence at a different radius. This coupling can cause non-local phenomena by local transport.

There are two typical radial propagations. One is the upstream propagation by increasing density at the plasma edge, triggered by the supersonic molecular beam injection (SMBI) and shallow pellet injection. The radial propagation is relatively slow with the time scale of confinement time. The other is the fast downstream propagation, so-called avalanche transport. Avalanche transport is characterized by the simultaneous radial propagation of a sharp gradient above the critical gradient and turbulence clouds. The propagation time scale is much shorter than the confinement time, and radial extension is much larger than the correlation length of the turbulence. Therefore, avalanche transport is local transport in the microscopic view but non-local transport in the macroscopic view.

The fluctuation intensity $I$ normalized to the kinetic energy density at diamagnetic drift velocity $v_{d i a}$ ) is given by (Hahm et al. 2004)

$$
\frac{\partial}{\partial t} I=\gamma_{L}(A) I-\gamma_{N L} I^{2}+D_{0} \frac{\partial}{\partial x} I \frac{\partial}{\partial x} I
$$

Here, $A=\nabla T_{a} / T$ denotes the temperature gradient, $D_{0}$ is the turbulent diffusion coefficient, $\gamma$ and $\gamma_{N L}$ are the linear growth rate and nonlinear damping rate, respectively. Figure 10 shows the time evolution of the temperature without turbulence spreading $\left(D_{0} I=0\right)$ and with turbulence spreading $\left(D_{0} I=1\right)$ (Hariri et al. 2016). The cold pulse is initiated at time $t=0$ by reducing the boundary value for the temperature by $20 \%$. The fast inward propagation of the cold pulse is visible in the outer plasma region. Cold pulse propagation slows somewhat toward the plasma center, 


\section{Non-local phenomena by radial propagation}

$\rho=\rho_{1}$

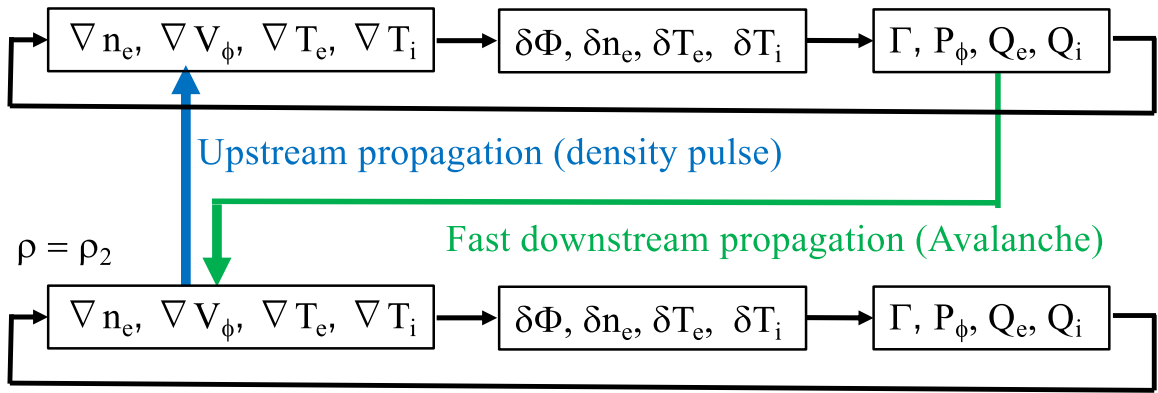

Slow upstream propagation

(density pulse propagation)

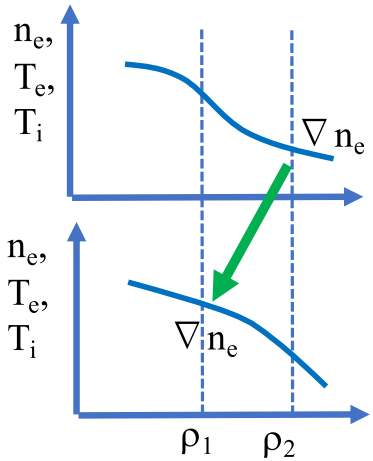

Fast downstream propagation (Avalanche transport)

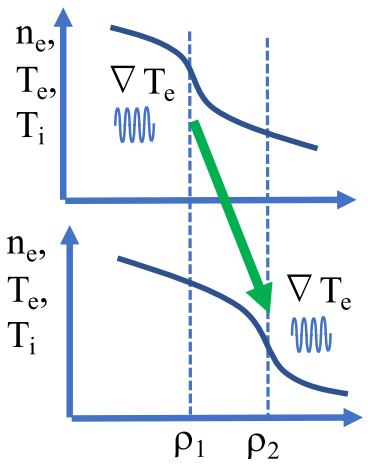

Fig. 9 Non-local phenomena by radial propagation

(a)

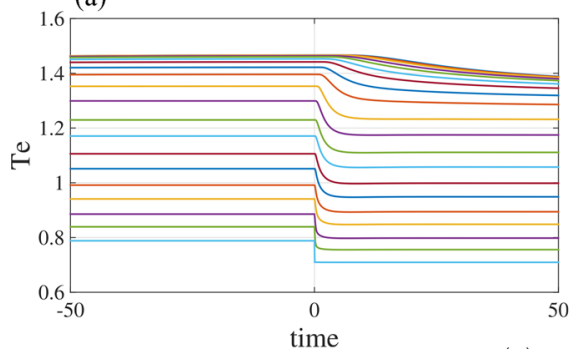

(b)

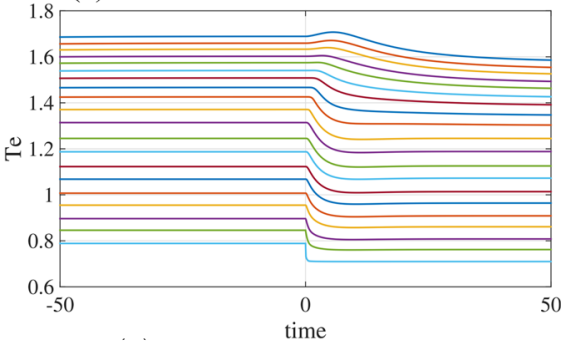

Fig. 10 Time evolution of temperature $\mathbf{a}$ without and $\mathbf{b}$ with turbulence spreading in the unit of keV. Here the cold pulse is triggered at $t=0$ (from Figs. 7 and 8 in (Hariri et al. 2016)) 
and the central temperature begins to drop after a few time units. In contrast, the central temperature increases after a few time units, which is called reversal of the cold pulse when turbulence is spreading $\left(D_{0} I=1\right)$. In this simulation, no reversal of the cold pulse is observed in the absence of turbulence spreading $\left(D_{0} I=0\right)$. This simulation demonstrates that turbulence spreading is necessary to reproduce the transient temperature rise in the cold pulse experiment. The non-local simulation predicts the transient temperature rise both in ion and in electron temperature. However, the local transport simulation described in Fig. 5 predicts the temperature rise only in electron temperature and ion temperature even decreases after the edge cooling by the cold pulse. Therefore, the temperature rises both in electron and ion temperature observed in the Alcator C-mod and LHD in Fig. 6 are clear evidence for the existence of non-local transport. Although the propagating non-local phenomena such as a cold pulse experiment can be explained by the radial propagation of fluctuation intensity $I$, the global non-local phenomena such as see-saw phenomena would need another mechanism.

\subsubsection{Avalanche}

An avalanche is the transport process caused by discrete, intermittent, and uncorrelated events and differs from so-called diffusive transport caused by smooth, steady, and correlated turbulence. Although the avalanche is a different class of processes that can contribute to transport, it is not easy to distinguish it from the diffusion process in an experiment. This is because the turbulence study based on the frequency domain requires a time window much larger than the time scale of the avalanche. The avalanche event appears as turbulence with a broadband spectrum in the fast Fourier transform (FFT) analysis, which is usually applied to improve the signalto-noise ratio. Therefore, the avalanche is masked by the usual turbulence unless the avalanche amplitude significantly exceeds the levels of smooth and steady turbulence (Kin et al. 2021).

Figure 11a shows an example of an avalanche event of electron temperature observed in ECE measurements in DIII-D tokamak (Politzer et al. 2002). Here, each curve is displaced vertically by an amount proportional to the normalized minor radius for that channel, as indicated by the left ordinate scale. It is not easy to detect avalanche events from a single curve because the amplitude of avalanche is comparable to the amplitude of turbulence. The avalanche events can be detected from the radial propagation of temperature perturbation. The highlighted bands indicate examples of avalanche events, and the radial velocity of this event is $\sim 300 \mathrm{~m} / \mathrm{s}$.

The wavelet analysis was applied to ECE data to evaluate the radial velocity of avalanche events. Figure $11 \mathrm{~b}$ shows the radial velocity as a function of radial position $(\rho)$ and the time scale of wavelet analysis. The avalanche events with a shorter time scale (high-frequency event) propagate faster than the avalanche events with a longer time scale (low-frequency event). The positive radial speed stands for the outward propagation of avalanche events, and the negative radial speed stands for the inward propagation. Interestingly, the inward propagation appears near the plasma center $(\rho<0.2)$, and its radial speed is smaller than the 

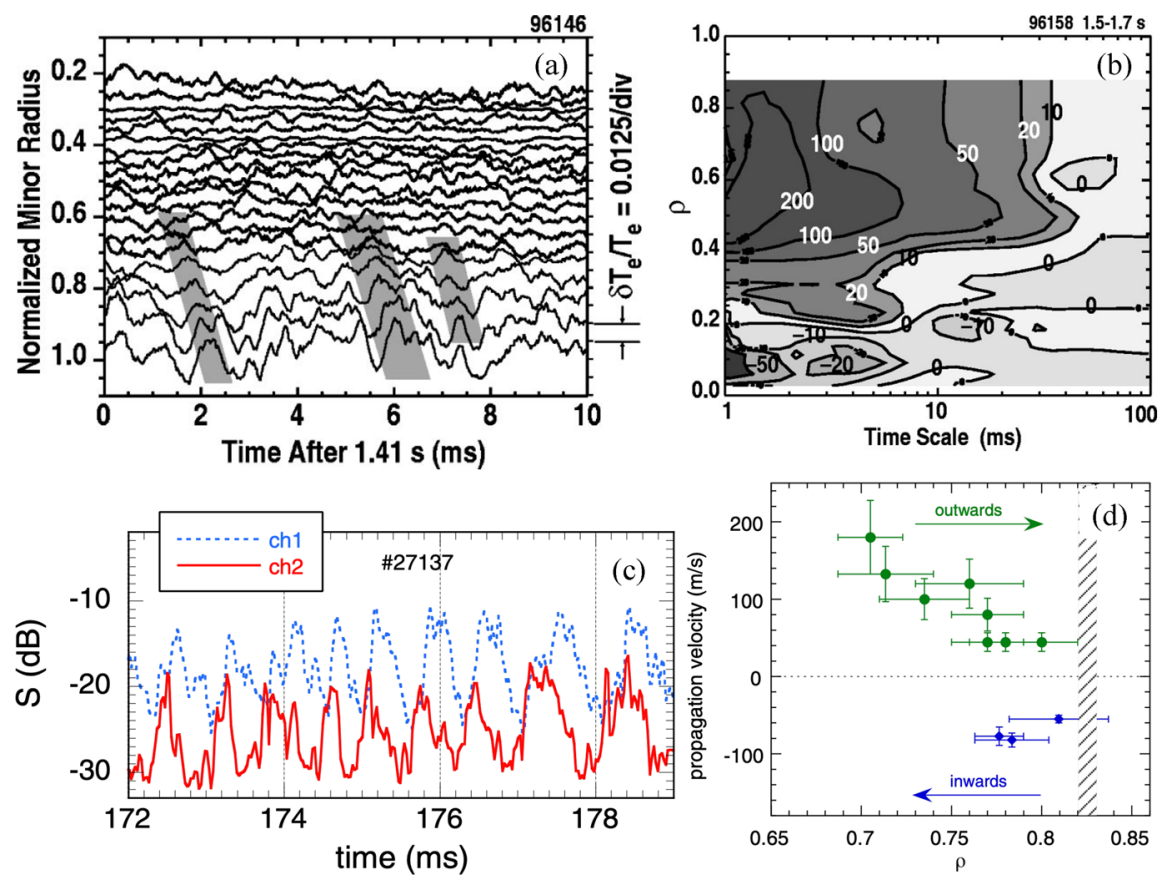

Fig. 11 a Time evolution of normalized electron temperature fluctuation and $\mathbf{b}$ radial velocity of avalanche events in unit of $\mathrm{m} / \mathrm{s}$ and $\mathbf{c}$ time evolution of density fluctuation level at channel 1 and 2 separated by $\delta \rho=0.05$ and $\mathbf{d}$ radial profiles of radial propagation velocity of density fluctuation. (from Figs. 4 and 11 in (Politzer et al. 2002) and from Figs. 4a and 5 in (Estrada et al. 2011a))

radial speed of outward propagation. The avalanche events originated at $\rho=0.2$ have bi-direction propagation both outward and inward.

Figure 11c, d shows time evolution of the density fluctuation level at channel 1 and channel 2 separated by $\delta \rho=0.05$ ( $\rho=0.75$ and 0.8$)$ and radial profiles of radial propagation velocity of the density fluctuation. Here the positive velocity stands for outward propagation, and negative velocity stands for inward propagation. The outward propagation velocity decreases as the $E \times B$ shear location (at $\rho=0.82$ ) is approached. Inward propagation velocities are represented using small symbols (in blue). The striped area indicates the radial location of the $E \times B$ shear. The outward propagation velocity observed in this experiment is $50-200 \mathrm{~m} / \mathrm{s}$ and comparable to the propagation of temperature perturbation observed in the DIII-D experiment. The flip of a sign of radial propagation direction of the density fluctuation is due to the transitioning role of the boundary between a suppressor (for the outward propagation case in a low-density regime) and a source (for the inward propagation case in a high-density regime). The increased density gradient may cause a transition of dominant micro-instabilities, which have different damping/driving mechanisms and effects on transport. This would be quite an interesting result because it de monstrates that the pedestal can 
be a source of turbulence spreading due to the steep pressure gradient and can be a sink of turbulence spreading due to the $E \times B$ shear. The inward propagation of density fluctuation and steep density gradient is observed in the limit cycle oscillation (LCO) that precedes an L-to-H transition (Kobayashi et al. 2013, 2014). This experiment would be another example of the plasma periphery region being a source of turbulence until the $E \times B$ shear becomes large enough to suppress the turbulence produced in the pedestal region.

The avalanche events observed in the experiment described above are characterized by the transient localized increase of temperature $(\delta T>0)$. More recently, the pair creation of bump $(\delta T>0)$ and void $(\delta T<0)$ has been experimentally identified in the KSTAR (Choi et al. 2019). After the pair creation, the void propagates inward, while the bump propagates outward. The bi-directional radial propagation of a void and a bump are attributed to the joint reflection symmetry in a self-organized critical system (Diamond and Hahm 1995), which is a different mechanism of the avalanche. The bi-directional radial propagation of the void and the bump is predicted by gyrokinetic full-f simulation, as seen in Fig. 12 (Kikuchi and Azumi 2012). The simulation reproduces the discrete, intermittent, radial propagation of the ion heat diffusivity (or ion temperature gradient) and radial electric field in a time scale much smaller than the ion-ion collision time $\left(\tau_{i i}\right)$. The simulation results show the bi-direction (outward and inward) propagation of temperature gradient and radial electric field $\left(E_{r}\right)$ shear from the normalized minor radius, $r / a$, of 0.6 (Idomura et al. 2009; Jolliet and Idomura 2012). The propagation direction of avalanches is changed depending on the sign of $E_{r}$ shear. In this simulation $E_{r}$ shear is negative $\left(\mathrm{d} E_{r} / \mathrm{d} r<0\right)$ at $r / a<0.6$ and positive $\left(\mathrm{d} E_{r} / \mathrm{d} r>0\right)$ at $r / a>0.6$.

Since the radial electric field, $E_{r}$, (and $E_{r}$ shear) is correlated with the temperature gradient (and the second derivative of temperature), the propagation of $E_{r}$ shear is accompanied by the propagation of bump with a negative second derivative and void with a positive second derivative. As illustrated in Fig. 12b, the bi-directional propagation of temperature perturbation can be understood as the bump and void propagations driven by the front with a large temperature gradient $(\mathrm{d} T / \mathrm{d} r)$ above the critical value where the turbulence is nonlinearly excited. Since many avalanche

(a)

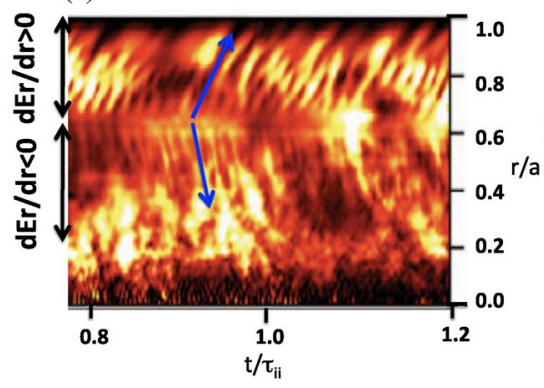

(b)

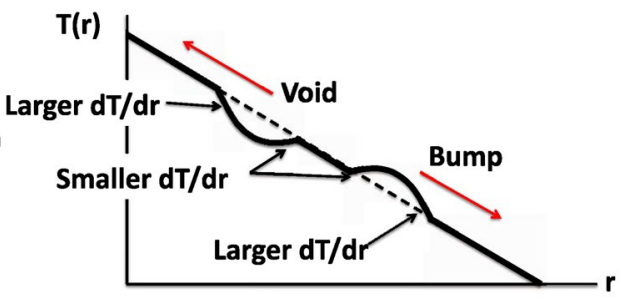

Fig. 12 a The spatio-temporal evolutions of the ion heat diffusivity calculated by Gyrokinetic full- $f$ simulation for positive $E_{r}$ shear and negative $E_{r}$ shear region and $\mathbf{b}$ bump and void avalanches near marginal stability (from figure 60 and figure 62 in (Kikuchi and Azumi 2012)) 
events $\left(\sim 10^{2}\right)$ occur within the ion-ion collision time, the macroscopic effect of avalanche events is usually observed in experiment.

\subsection{Mediator}

The role of the mediator of turbulence in non-local phenomena is illustrated in Fig. 13. The mediator of turbulence is crucial to understand the non-local phenomena because the correlation length of micro-turbulence is too short to cause the radial coupling of turbulence. The mediator should have a long correlation and nonlinear interaction with micro-turbulence. The energy transfer from micro-turbulence to the mediator at one location and from the mediator to micro-turbulence at a different location is essential for radial coupling. The candidates for the mediator of turbulence are meso/macro-scale turbulence, MHD fluctuation, and zonal flow.

It is difficult to distinguish the turbulence driven by a non-local mediator from the locally driven turbulence in a steady-state condition. Therefore, the cross bi-coherence between the fluctuation of the mediator with low-frequency and high-frequency turbulence is experimental evidence of a non-local mediator. The experimental results of bi-coherence for macro-scale (long-range) fluctuations, MHD instability, and a zonal flow non-local mediator are summarized in Fig. 14. Figure 14a, b

\section{Non-local phenomena by mediator of turbulence}
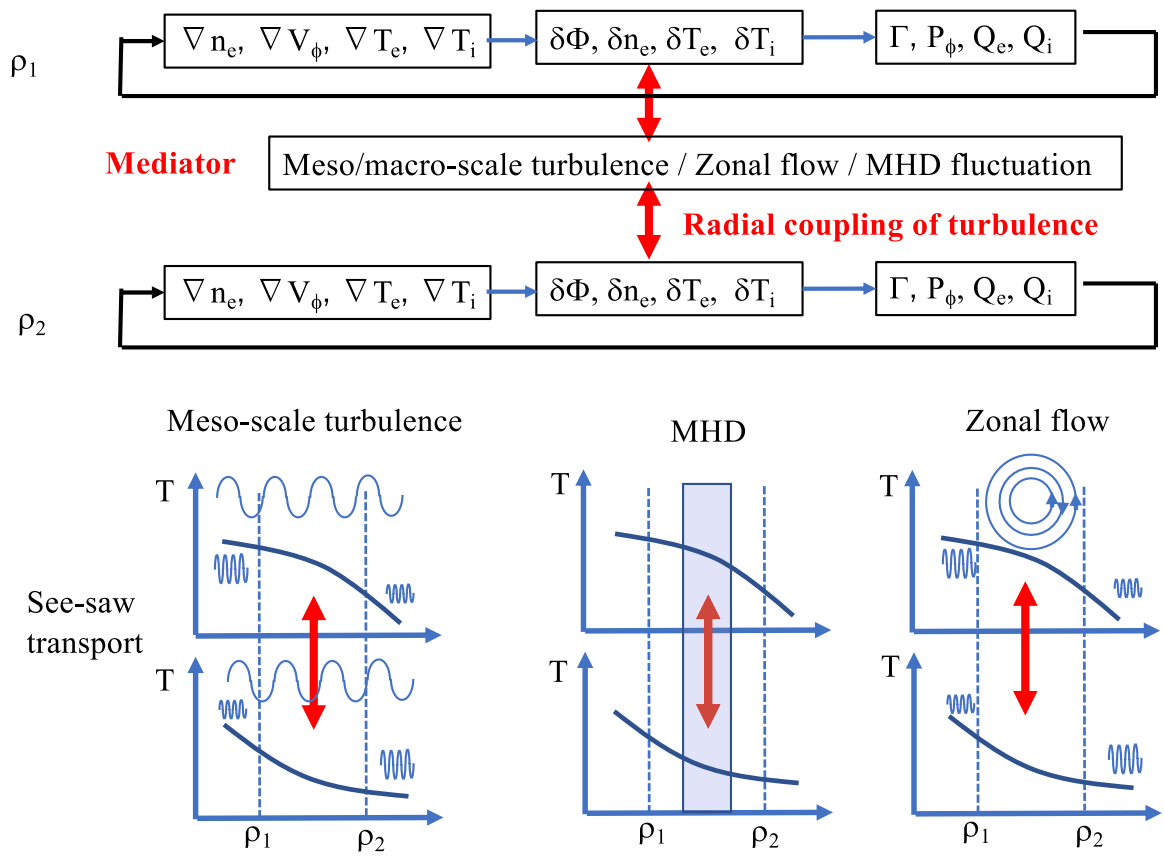

Fig. 13 Non-local phenomena by mediator of turbulence 


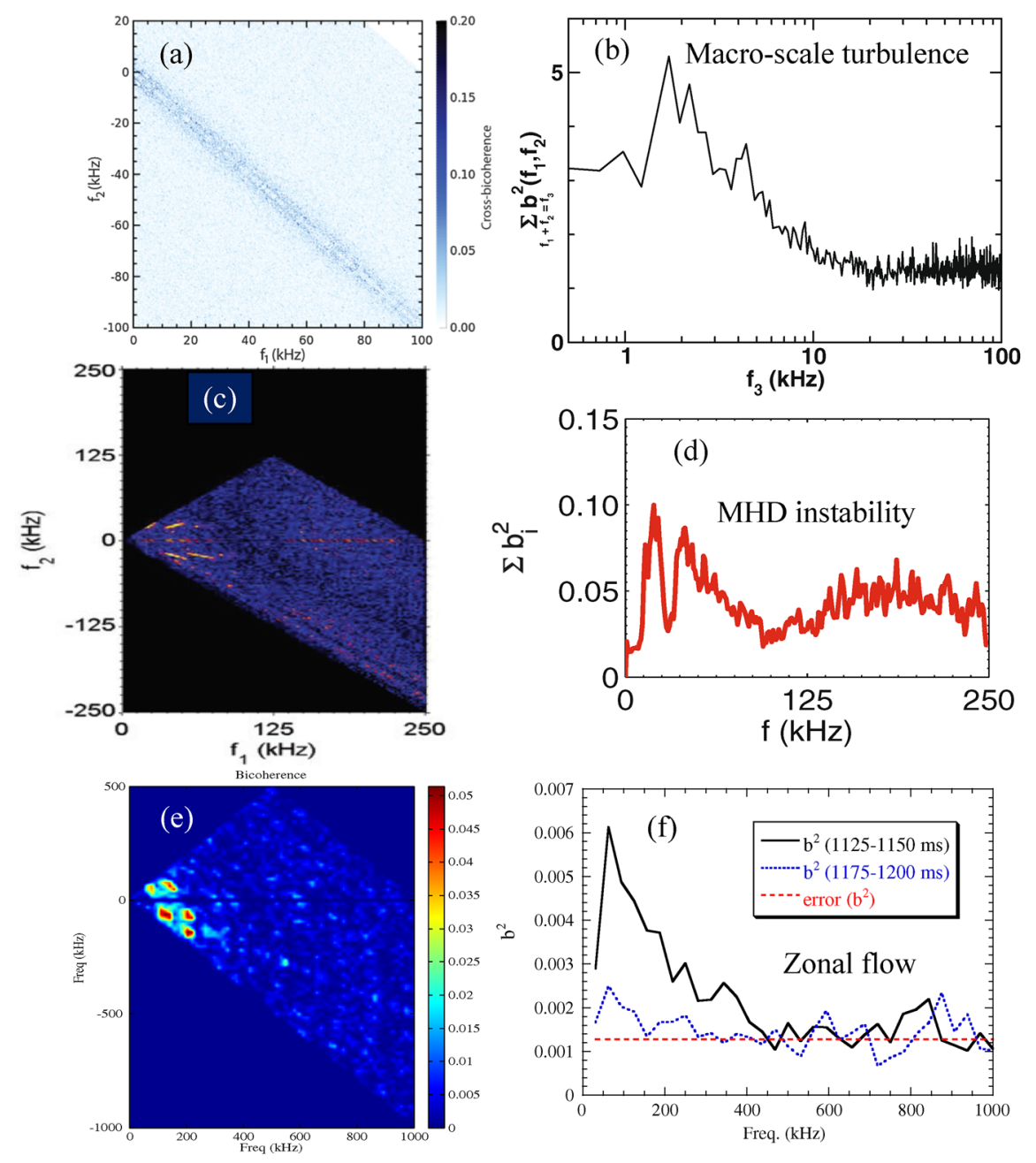

Fig. 14 a Cross-bi-coherence between density and temperature fluctuations, $\mathbf{b}$ total cross bi-coherence for fixed $f_{3}$ for long-range fluctuations, c cross bi-coherence, $\mathbf{d}$ total bi-coherence for MHD instability, e auto bi-coherence during biasing $(1125<t<1150 \mathrm{~ms})$ and $\mathbf{f}$ total bi-coherence during and after biasing $(1175<t<1200 \mathrm{~ms}$ ) for zonal flow (from Fig. 4 in (Inagaki et al. 2011) and Fig. 4 in (Inagaki et al. 2012a), from Fig. 5d, h in (Chen et al. 2016), from Figs. 4 and 5 in (van Milligen et al. 2008))

shows cross bi-coherence between density and temperature fluctuations, total cross bi-coherence for fixed $f_{3}$ for long-range fluctuations in the ECH heated plasma in the LHD (Inagaki et al. 2011). The existence of the cross bi-coherence between macroscale fluctuation $(1.7 \mathrm{kHz})$ and micro-scale density fluctuation $(<100 \mathrm{kHz})$ also supports that the macro-scale fluctuation plays the role of a non-local mediator. Figure 14(c)(d) shows cross bi-coherence between temperature fluctuations and magnetic field fluctuations driven by fishbone MHD instability in HL-2A (Chen et al. 2016). The clear frequency peak of the cross bi-coherence spectrum was observed 
with a fishbone frequency at $\sim 20 \mathrm{kHz}$. Figure 14e, f shows auto bi-coherence during biasing $(1125<t<1150 \mathrm{~ms})$ and total bi-coherence during and after biasing $(1175<t<1200 \mathrm{~ms})$ measured with a Langmuir probe in the TJ-II stellarator (van Milligen et al. 2008). Bi-coherence with a peak of low frequency around $10 \mathrm{kHz}$ was observed. (This low frequency is not well resolved in this figure, but it is deduced from a high-resolution Fourier spectrum, calculated in the biasing time interval). The biasing produces a sharp increase in coherence between $E_{\theta}$ and $E_{r}$ in the frequency range $100<f<250 \mathrm{kHz}$ and also at a low frequency (possibly related to the shear or zonal flow frequency).

Bi-coherence analysis was applied for three waves with the frequency of $f_{1}, f_{2}, f_{3}$ (Inagaki et al. 2012a) with a matching condition of $f_{1}+f_{2}=f_{3}$. The bi-coherence analysis is widely used to study the nonlinear interaction between two waves. Since the temperature fluctuation with a high-frequency region is dominated by noise, it is difficult to identify nonlinear interactions between the long-range and microscopic fluctuations by auto bi-spectrum analysis. The cross bi-coherence between temperature and density $(1-100 \mathrm{kHz})$ measured with a reflectometer was analyzed. The clear frequency peak of the cross bi-coherence spectrum was observed at $1.7 \mathrm{kHz}$. Since the ensemble $(N)$ is limited in the experiment, the convergence study was also applied to cross bi-coherence analysis (Nagashima et al. 2006). In the convergence study, the finite cross bi-coherence value at $\mathrm{N} \rightarrow \infty$ is checked. If the cross bi-coherence is dominated by noise, the bi-coherence value for $\mathrm{N} \rightarrow \infty$ becomes zero. The cross bi-coherence value for $f_{3}=1.7 \mathrm{kHz}$ has clear finite value at $1 / \mathrm{N}=0$. These results are clear evidence for the nonlinear coupling between the long-range fluctuations and the microscopic fluctuations. Although the macro-scale fluctuation has only a small contribution to the enhancement of radial flux due to the low frequency, it can play an important role in the energy transfer between the micro-scale turbulence at a different location and can be a main player for non-local transport (Inagaki et al. 2014).

\subsubsection{Macro-scale turbulence}

Macro-scale electron temperature fluctuation observed in the LHD has a long-distance radial correlation (Inagaki et al. 2011; Xu et al. 2011; Inagaki et al. 2012a, b). This macro-scale fluctuation plays the role of mediator of two micro-scale turbulence excited at different plasma radii. Figure $15 \mathrm{a}-\mathrm{e}$ shows the time evolution of low-frequency electron temperature fluctuation with long-range correlation $(\rho=0.12-0.58 \mathrm{e})$. The radial correlation length is comparable to the minor plasma radius. The frequency of this temperature fluctuation is $\sim 1-3 \mathrm{kHz}$, which was previously considered to be MHD oscillation. However, this temperature fluctuation has various characteristics different from the usual MHD oscillation. It has a phase delay in the radial direction, which indicates the spiral structure of fluctuation. The amplitude of the magnetic field is much smaller than that predicted by the displacement of the magnetic flux surface, evaluated from temperature fluctuation and temperature gradient.

Figure $15 \mathrm{f}-\mathrm{i}$ shows modulated ECH power, a conditional averaged temperature gradient, the amplitude of long-range density fluctuations $(1.5-3.5 \mathrm{e} \mathrm{kHz})$, and the 

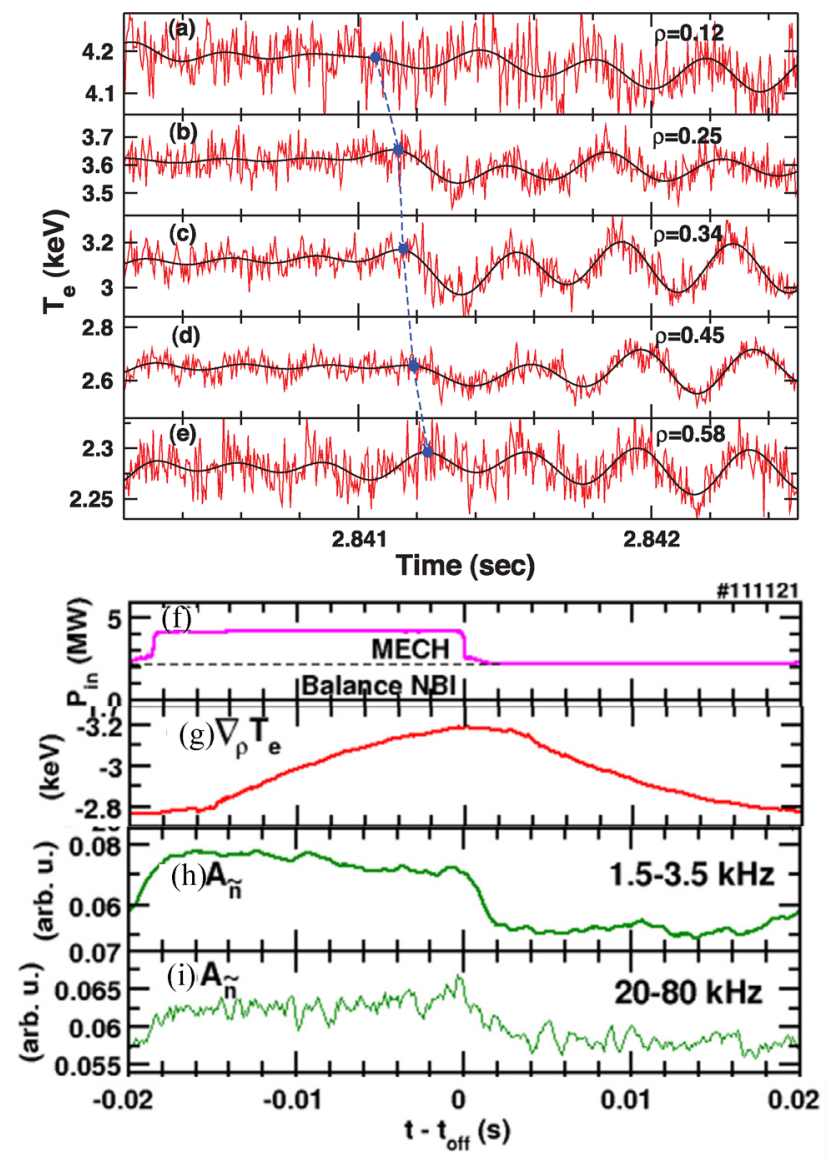

Fig. 15 a-e Time evolution of electron temperature fluctuation (red line) and low-pass filtered $(\leq 3.5$ $\mathrm{kHz}$ ) signal (black line) at abrupt change of fluctuation amplitude. Time evolution of $\mathbf{f}$ heating power of modulated ECH, $\mathbf{g}$ temperature gradient, $\mathbf{h}$ amplitude of long-range density fluctuations (1.5-3.5e $\mathrm{kHz}$ ), and $\mathbf{i}$ amplitude of micro-scale density fluctuations outside $\mathrm{ECH}$ deposition area at $\rho=0.63$. (from figure 5 in (Inagaki et al. 2011) from Fig. 1a, e, g, h in (Inagaki et al. 2013))

amplitude of micro-scale density fluctuations $(20-80 \mathrm{kHz})$ outside the ECH deposition area at $\rho=0.63$ in the LHD (Inagaki et al. 2013). The increase of density fluctuation both for low and high frequency is rapid within $2 \mathrm{~ms}$, but the rise of the temperature gradient is slow $(\sim 20 \mathrm{~ms})$ and delayed $(\sim 5 \mathrm{~ms})$. Immediately after the onset of the ECH pulse (2-5 ms), the density fluctuation levels are already high, but the temperature gradient is still low. These results clearly show that a non-local process drives these density fluctuations. An increase of micro-turbulence and macroscale electron temperature fluctuation outside the heat deposition area during the modulation ECH observed strongly suggests that this macro-scale electron temperature fluctuation is a non-local mediator of micro-turbulence between the inside and outside of the heat deposition area. 
The hysteresis observed in the gradient-flux relation of the plasma with modulation ECH plotted in Fig. 7 can be interpreted by non-local transport by a mediator of this macro scale turbulence. The jump of heat flux is interpreted as an increase of heat flux before increasing the temperature gradient at the radius where heat deposition is negligible. The abrupt increase of heat flux without an accompanying increase of temperature gradient is due to enhanced turbulence, which is driven by the dynamical force in plasma phase space (Itoh and Itoh 2012) inside the region (at a smaller plasma radius, not at the radius of interest). The macro-scale turbulence plays the role of mediator in transferring the micro-turbulence driven inside the region to the outer area much faster than the heat pulse, which causes the increase of temperature gradient in the outer area.

\subsubsection{MHD instability}

The experiment in HL-2A demonstrated a nonlinear coupling between the fishbone and background turbulence (Chen et al. 2016). Then, MHD instability was also recognized as a candidate as a mediator of turbulence that causes non-local transport phenomena. Figure 16 shows the time evolution of the electron temperature, $T_{e}$, at $\rho=0.23$ and 0.83 , and the Mirnov signal, $\mathrm{d} B_{\theta} / \mathrm{d} t$, and its RMS, $\left\langle\delta B_{\theta}\right\rangle_{\mathrm{rms}}$. The Mirnov signal shows the repeated fishbone bursting. With the fishbone bursting and core $(\rho=0.23)$ heating a simultaneous decrease in electron temperature is observed at the plasma edge $(\rho=0.83)$. This simultaneous increase and decrease of temperature in the core and edge are see-saw phenomena, caused by non-local transport, discussed in section 3.3 .

Figure 16b, c shows the Lissajous figures in the space of change in electron temperature, $\Delta T_{e} /\left\langle T_{e}\right\rangle$, and root mean square (RMS) of the Mirnov signal $\left\langle\delta B_{\theta}\right\rangle_{\mathrm{RMS}}$. The rotation directions of Lissajous figures at $\rho=0.30$ are clockwise $(\mathrm{CW})$, while the rotation directions at $\rho=0.65$ are counter clockwise $(\mathrm{CCW})$. The opposite rotation directions of Lissajous figures inside and outside the fishbone excited region suggest that magnetic perturbations play a crucial role in non-local phenomena.

\subsubsection{Zonal flow}

Zonal flows are an azimuthally symmetric band like shear flows driven by drift wave turbulence (Fujisawa 2009). It was experimentally identified using two heavy ion beam probes (HIBP) in Compact Helical System (CHS) (Fujisawa et al. 2004). Anticorrelation between fluctuation amplitude and zonal flow amplitude supports the predator-prey model between the zonal flow and drift wave turbulence (Diamond et al. 1994; Kim and Diamond 2003; Diamond 2011). The predator-prey model was experimentally confirmed in the experiment (Itoh et al. 2007). Zonal flow is another candidate for the mediator of turbulence. Zonal flow driven by drift wave turbulence can suppress the drift wave turbulence. In other words, zonal flow grows, extracting energy from microscopic fluctuations to reduce turbulence and turbulent transport. Since the radial correlation length of zonal flow is longer than that for microscopic fluctuations, it can suppress fluctuations at a different radius, via induction of the zonal flow (Itoh et al. 2009). 


\section{Mechanism for non-local transport nature MHD}

(a)
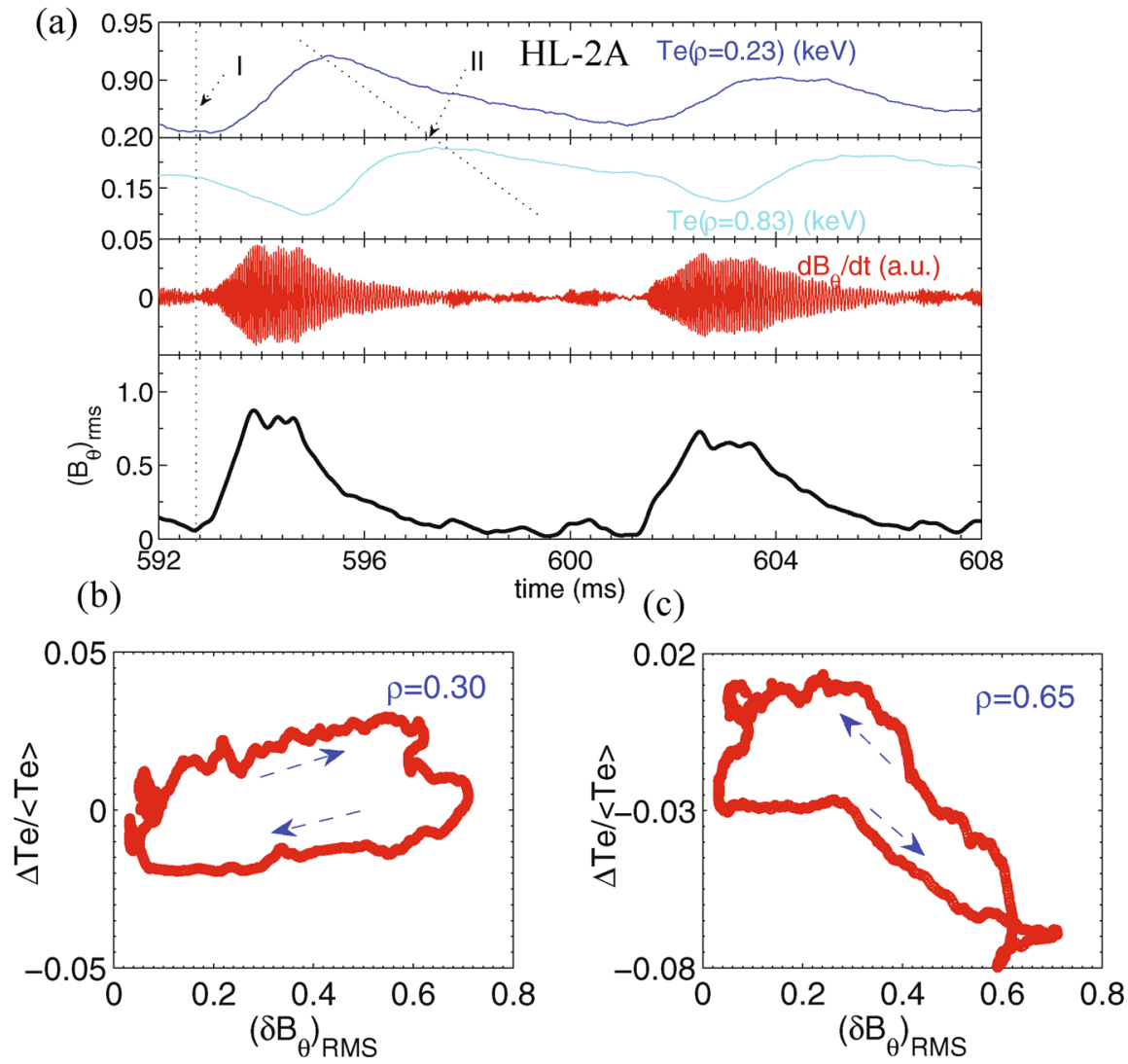

Fig. 16 a Time evolution of electron temperature at $\rho=0.23$ and 0.83 , and Mirnov signal and its RMS, and $\mathbf{b}$, $\mathbf{c}$ Lissajous figures between the change in normalized electron temperature and RMS of the Mirnov signal at $\rho=0.30$ and 0.65 (from Figs. 3 and 4 in (Chen et al. 2016))

The absolute rates of nonlinear energy transfer among broadband turbulence, lowfrequency zonal flows (ZFs) and geodesic acoustic modes (GAMs) were measured in HL-2A (Xu et. a., 2012). Figure 17a and b shows the auto-spectra of potential and perpendicular velocity fluctuations, respectively, at a position $\sim 2.5 \mathrm{~cm}$ inside the LCFS. As the ECH power increases from 0 to $730 \mathrm{~kW}$, low-frequency zonal flow (frequency $f<1-2 \mathrm{kHz}$ ) significantly grows, while the amplitude of Geodesic Acoustic Mode (GAMs) with a peak frequency of $10 \mathrm{kHz}$ is almost unchanged. This measurement indicates that much stronger zonal flow, especially the low-frequency type, developed as the temperature gradient increased. Figure $17 \mathrm{c}$ and d shows nonlinear kinetic energy transfer rates and effective growth rates. Although most of the turbulent kinetic energy is transferred to the large-scale shear flows, the turbulent energy with intermediate frequencies is also nonlinearly transferred to fluctuations 


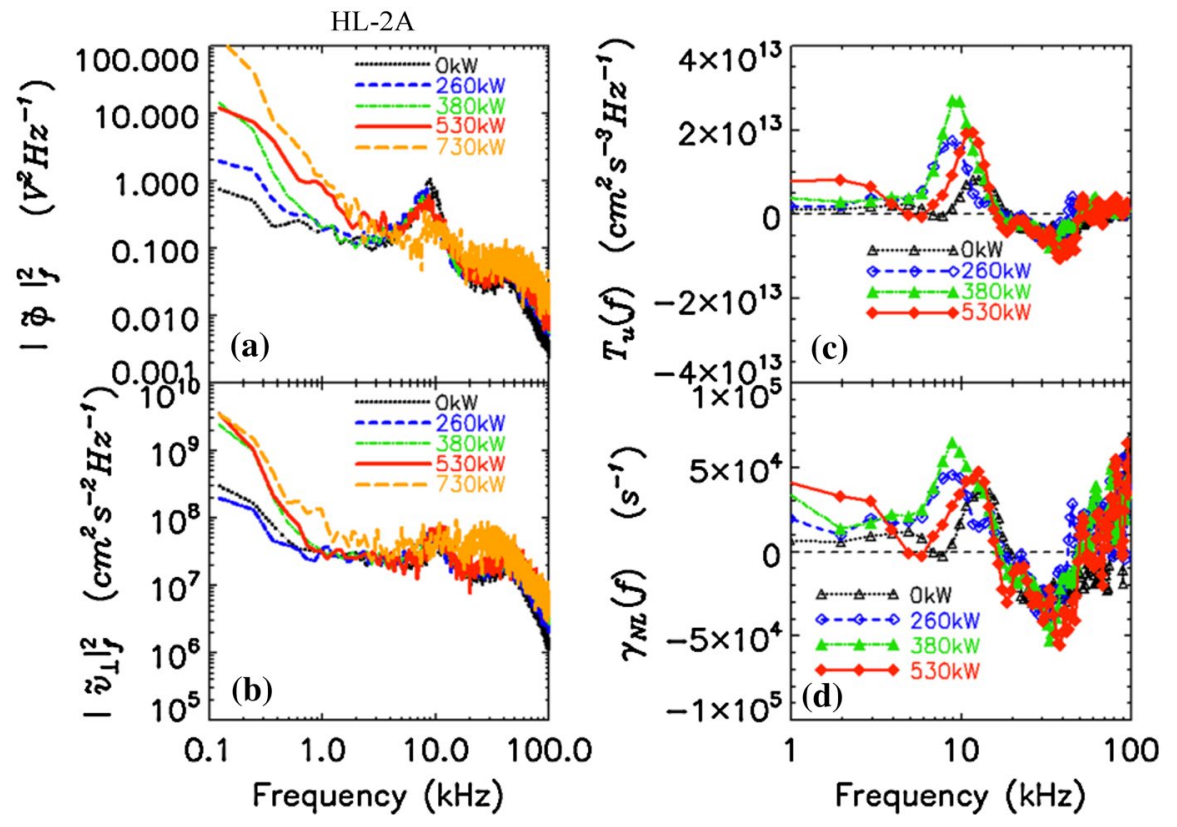

Fig. 17 Frequency spectrum of a potential fluctuation, b perpendicular velocity fluctuation, $\mathbf{c}$ nonlinear kinetic energy transfer rate, and $\mathbf{d}$ effective growth rate (from Fig. 2 in (Xu et al. 2012))

with higher frequencies $(f>80 \mathrm{kHz})$. This observation strongly supports the theoretical model of energy transfer between turbulence and zonal flow, essential for the mediator of turbulence to explain the non-local phenomena.

\section{Impact of non-local transport}

Non-local transport is essential to understand non-local phenomena at the transient phase in toroidal plasmas. However, non-local transport also has a substantial impact on structure formation (radial profile) in a steady state. The mechanism of non-local transport always exists in the plasma, both in the transient and steady-state phases.

Nonlinear coupling between micro-turbulence and meso-(or macro) turbulence causes turbulence spreading. The structure formation (density, temperature profiles) is strongly influenced by the turbulence spreading.

The examples of the structure formation are illustrated in Fig. 18. The turbulence spreading from the region outside ITB to the area inside ITB determines the sharpness of the ITB foot. The corrugation (so-called staircase) is a unique and interesting structure formation in toroidal plasmas. The turbulence spreading would be an important mechanism to determine the radial structure. The turbulence spreading would also be essential inside the magnetic island because no turbulence is excited inside the magnetic island due to the flattening of temperature and density. The 


\section{Impact of turbulence spreading on steady-state profile}

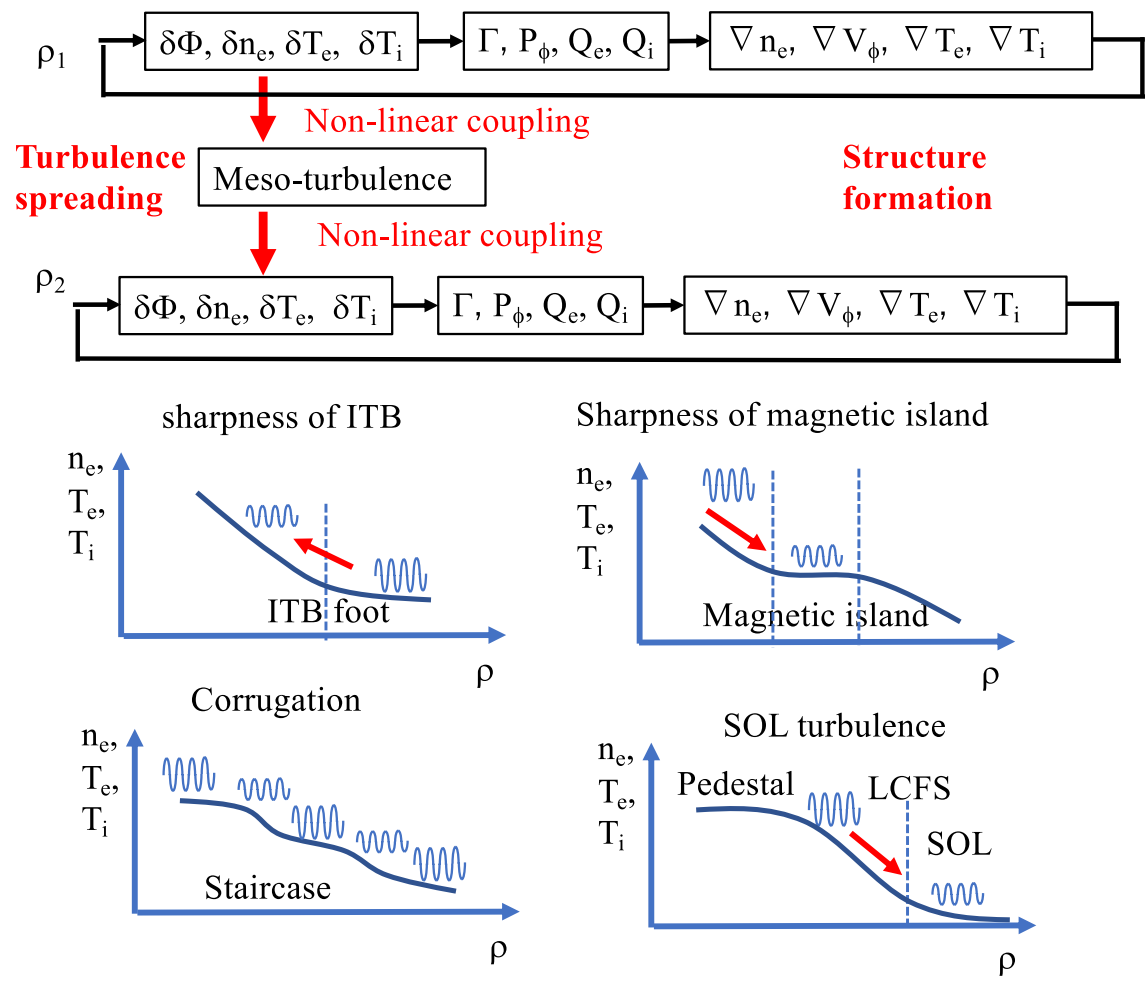

Fig. 18 Impact of turbulence spreading on steady-state profile

scrape-off layer (SOL) is also the region where no turbulence is excited, and spreading turbulence is dominant similar to the magnetic island.

\subsection{Turbulence spreading into the ITB region}

The internal transport barrier (ITB) is characterized by the radial temperature profile with a sharp gradient region, which appears in interior plasma (Ida and Fujita 2018a). In the ITB region, the turbulence is strongly suppressed (stable region), while the turbulence is enhanced (unstable region), outside the ITB region. As seen in Fig. 19, the micro-scale turbulence in the unstable region spreads into the stable region through the nonlinear coupling with mesoscale and macro-scale turbulence (Hahm et al. 2004; Gürcan and Diamond 2006; Hahm and Diamond 2018). When the turbulence is spreading weakly, the ITB has discontinuity of gradient, a so-called ITB foot. In contrast, this discontinuity disappears, and the ITB foot becomes unclear when the turbulence spreading becomes strong. Once the turbulence spreading occurs, the discontinuity of gradient (i.e. the second derivative of temperature) becomes weak and the $E \times B$ shear also becomes 
(a)

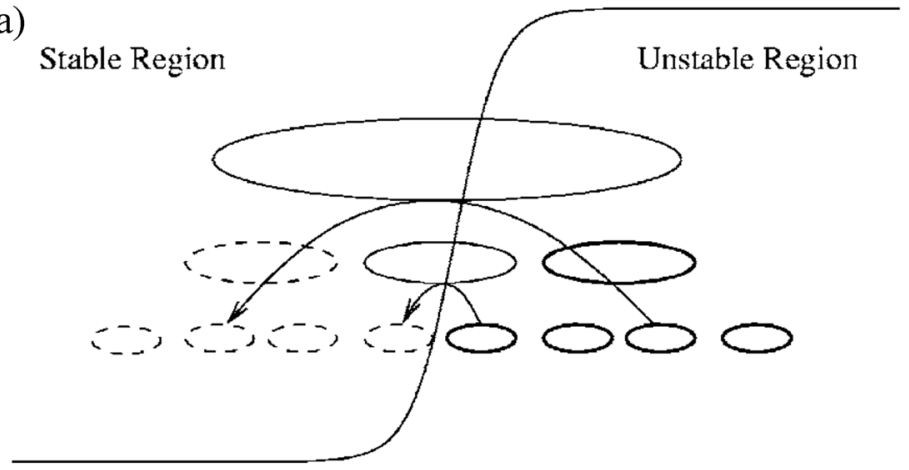

(b)

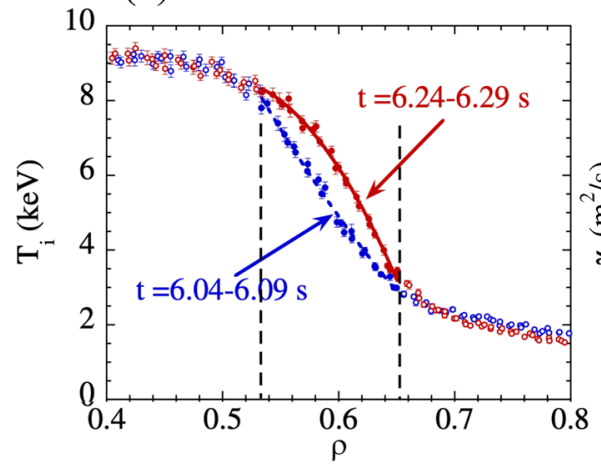

(c)

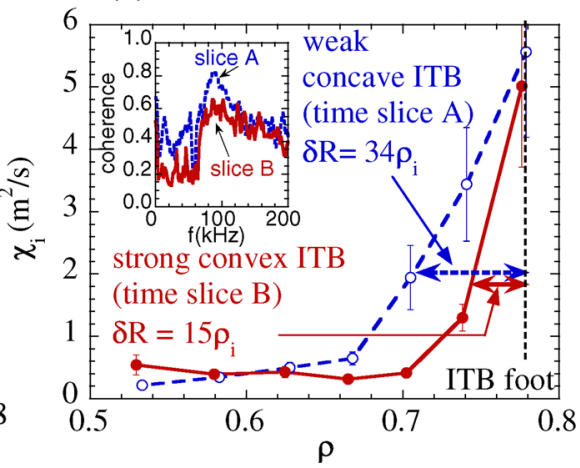

Fig. 19 a Diagram of turbulence spreading by nonlinear mode couplings and radial profiles of $\mathbf{b}$ ion temperature and $\mathbf{c}$ ion thermal diffusivity for weak concave ITB $(t=6.04-6.09 \mathrm{e}$ s: time slice A) and strong convex ITB ( $t=6.24-6.29 \mathrm{e}$ s: time slice B). (from Fig. 1 in (Gürcan and Diamond 2006) and Fig. 1a and Fig. 4 in (Ida et al. 2008))

weak. Since the $E \times B$ shear contributes to the block of turbulence spreading, further instances of it occur. This feedback process causes the bifurcation of the ITB structure with and without a clear foot point.

As seen in Fig. 19b, c, the bifurcation of the ITB structure was observed in JT-60U (Ida et al. 2008). This is called curvature bifurcation of the ITB. One is a concave ITB with a clear shoulder structure but no foot structure $(t=6.04$ $-6.09 \mathrm{e} s)$. The other is a convex ITB with a clear foot structure but no shoulder structure $(t=6.24-6.29 \mathrm{e} \mathrm{s})$. The ITB structure alternates between a concave ITB and a convex ITB for the steady-state heating condition. The concave ITB has a gradual decrease of ion thermal diffusivity near the ITB foot, while the convex ITB has a sharp drop of ion thermal diffusivity near the ITB foot. The decay length is 34 times that of ion gyroradius $\left(\rho_{i}\right)$ for the concave ITB and 15 times that of ion gyroradius for the concave ITB. The longer decay length indicates a deeper penetration of turbulence due to the stronger turbulence spreading, consistent with a larger coherence of the turbulence, measured just inside the ITB region, with a separation of $14 \rho_{i}$ at time slice A. 
The other important mechanism determining the radial structure in the plasma with the ITB is non-local transport by mediators. The most significant impact of non-local transport by mediators is the core-edge coupling of transport, where the core and edge transport changes simultaneously. This is observed as the enhancement of edge transport when the core transport is reduced by the formation of the ITB or as the reduction of edge transport when the increase of the $T_{e} / T_{i}$ ratio enhances the core ion transport by applying ECH as discussed in Fig. 8.

\subsection{Pressure corrugation with $E \times B$ staircase}

The $E \times B$ staircase is a structure formation characterized by a spontaneously formed, self-organizing pattern of quasi-regular, long-lived, localized $E \times B$ shear flow (Dif-Pradalier et al. 2015, 2017; Qi et al. 2019). This coincides with long-lived pressure corrugations and interacting avalanches, as illustrated in Fig. 20. Turbulence spreading plays a crucial role in structure formation. Finite turbulence spreading is necessary to smooth the staircase structure's curvature at the corners of the jump and step. However, the enhancement of turbulence spreading tends to wash out the pattern (Guo et al. 2019). Mesoscale transport events, such as avalanches or turbulence pulses (i.e., spreading), drive inhomogeneous mixing and transport of potential vorticity. The inhomogeneous mixing produces corrugations and $E \times B$ shear layer. This process is a mechanism for zonal profile corrugations and staircase formation (Leconte and Kobayashi 2021).

(a)

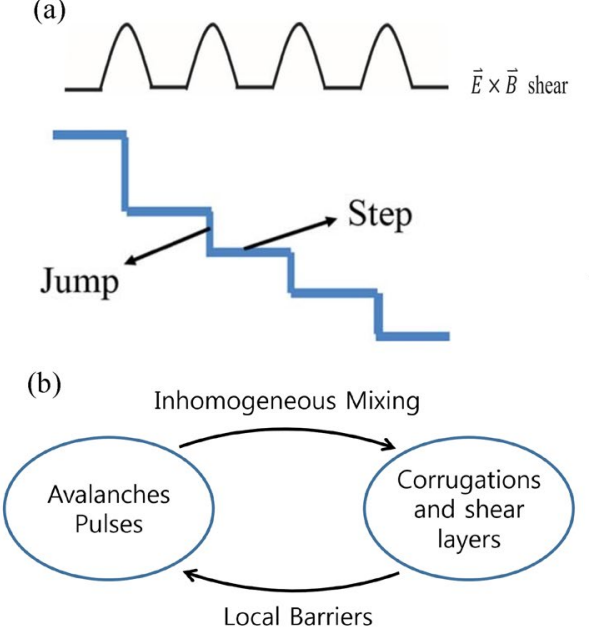

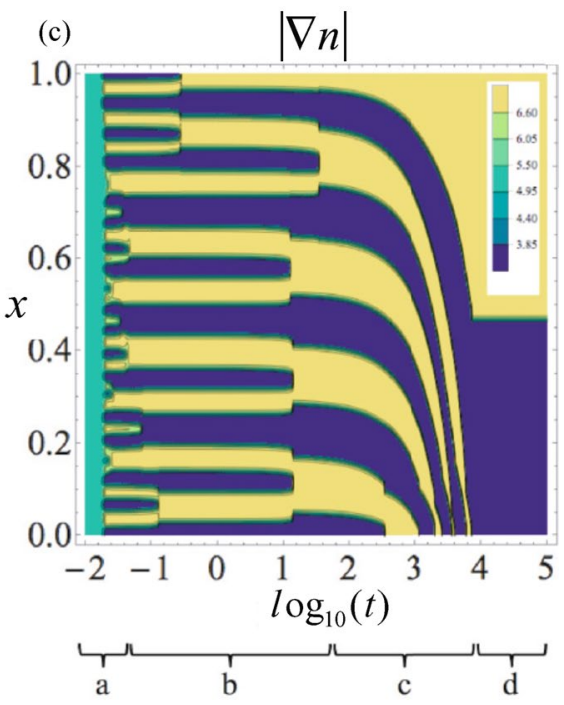

Fig. 20 a $E \times B$ staircase and pressure profile, $\mathbf{b}$ diagram of local barrier formation by inhomogeneous mixing, and contour plot of the time evolution of density gradient at the condensation of staircase structure. The horizontal axis is the log of time, and the vertical axis is the scaled radius. (from Fig. 1 and Fig. 4 in (Guo et al. 2019) and figure 59 in (Hahm and Diamond 2018) and Fig. 1 in (Ashourvan and Diamond 2016)) 
These corrugations contribute to the formation of local barriers and drive avalanches or turbulence pulses, as seen in Fig. 20. Because of this feedback loop, a spontaneous development of structure can cause a condensation of the staircase structure (Ashourvan and Diamond 2016). Figure 20c is an example of staircase structure condensation of electron density. The density staircase structure develops into a lattice of mesoscale jumps and steps. The jumps then merge and migrate in radius, leading to a new macro-scale profile structure. As seen in the time evolution of density gradient in space, many corrugations are seen initially. Then, however, these corrugations merge into each other and finally produce the macrostructure with enhanced confinement.

\subsection{Turbulence spreading into the magnetic island}

A magnetic island is a closed magnetic flux surface bounded by a separatrix (X-point), isolating it from the rest of the space. Since the radial heat flux perpendicular to the magnetic flux surface flows through the X-point, the temperature profile becomes almost flat (nearly zero temperature gradient) at the O-point of the magnetic island in the steady state. The O-point of the magnetic island becomes a stable region because the gradient is too small to generate turbulence. Therefore, the turbulence observed inside the magnetic island should be not driven turbulence but the spreading turbulence propagated from outside the magnetic island. It is an interesting question where the turbulence spreading occurs around the boundary of the magnetic island (X-point poloidal angle of O-point poloidal angle). The $E \times B$ shear layer, which contributes to the block of turbulence spreading, is weak near the $\mathrm{X}$-point (Hahm et al. 2021). The X-point of the magnetic island is the possible root for turbulence spreading.

The heat pulse propagation experiment using modulated ECH in DIII-D demonstrates that the turbulence spreading occurs through the X-point of the magnetic island. Figure 21 shows the radial profiles of normalized density fluctuation, modulation amplitude of density fluctuation, and the delay time of density fluctuation modulation with respect to the temperature modulation (Ida et al. 2018b). Here positive delay time means that the temperature rise is earlier than fluctuation amplitude rise, while minus delay means that the fluctuation amplitude rise occurs before the temperature rise. The delay time is positive at the X-point but is negative in the outer half of the magnetic island at the O-point poloidal angle. Since the turbulence level at the O-point region is much lower than that at the $\mathrm{X}$-point region, the heat pulse gradually propagates from the boundary of the magnetic island to its O-point. The time scale of this propagation is a few milliseconds to tens of milliseconds. This negative delay time (the earlier arrival of the density fluctuation pulse) is definite evidence for the turbulence spreading from the $\mathrm{X}$-point to the $\mathrm{O}$-point along the poloidal direction.

The bifurcation phenomena due to the interplay between turbulence spreading and the $E \times B$ shear layer can occur similarly to the case of the ITB foot discussed in 5.1. Figure 21(c) shows the bifurcation of phenomena observed in the modulation decay length of a heat pulse (Ida et al. 2015b). The modulation amplitude of the heat 

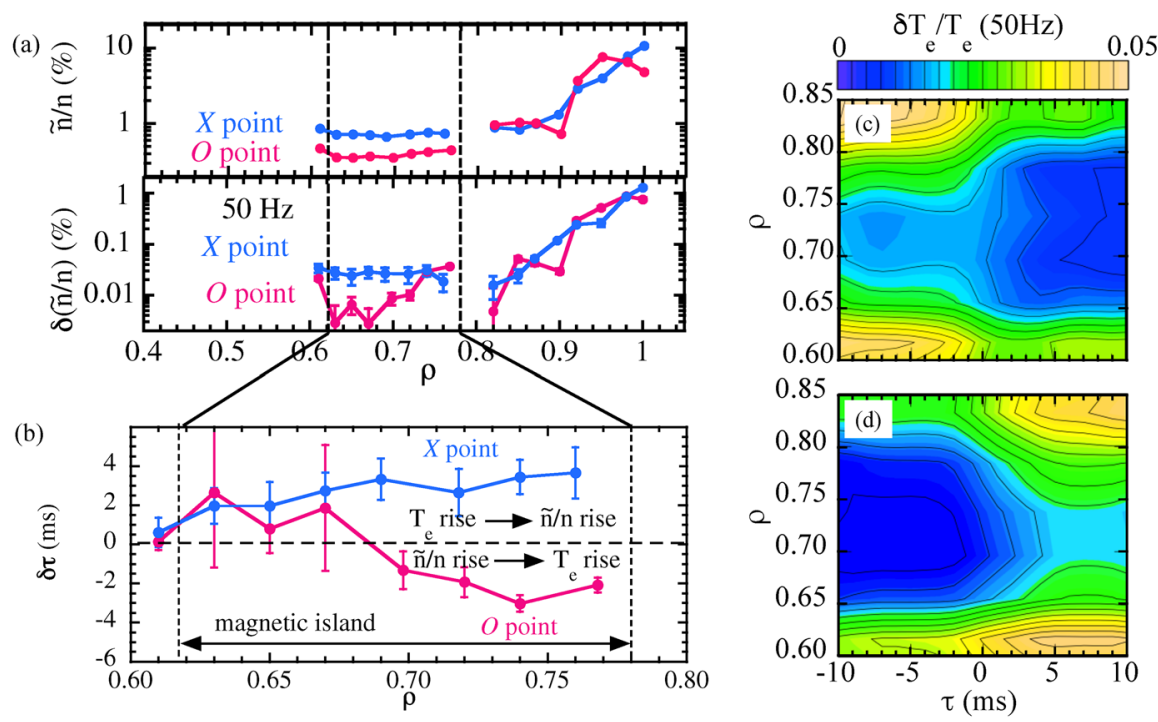

Fig. 21 a Radial profiles of normalized density fluctuation, its modulation amplitude and b radial profile of delay time difference between modulation of electron temperature and modulation of normalized density fluctuation at X-point and O-point of magnetic island. Contour of relative modulation amplitude of electron temperature in space and time during the $\mathbf{c}$ forward transition (from high accessibility to low accessibility magnetic island) and $\mathbf{d}$ backward transition (from low accessibility to high accessibility magnetic island) at O-point of magnetic island (from Figs. 2 and 3 in (Ida et al. 2018b) and Fig. 6 in (Ida et al. 2015b))

pulse is roughly in inverse proportion to the heat pulse propagation speed into the magnetic island (Ida et al. 2016). As the pulse propagation speed from the boundary to the O-point is slow, the amount of heat pulse to the O-point of the magnetic island becomes small, and most of it propagates through the X-point. The transition from the state with a longer decay length (deep penetration of the heat pulse) to the state with a shorter decay length (shallow penetration of the heat pulse) occurs within a time scale of few milliseconds. The former state is called a high accessibility state, and the latter state a low accessibility state. The back transition from the low accessibility state to the high accessibility state is also observed. Therefore, the transition of the modulation decay length indicates the bifurcation of turbulence spreading. The stochastization of the magnetic field at the X-point of the magnetic island is a key for the bifurcation of turbulence spreading into the magnetic island. A slight increase of stochastization magnitude weakens the $E \times B$ flow shear and enhances the turbulence spreading.

The X-point stochastization plays the role of a valve for turbulence spreading into the magnetic island (Ida 2020). The penetration of heat pulse characterizes two metastable states, depending on opening/closing this valve. Deep penetration of heat pulse occurs due to the significant turbulence spreading with low $E \times B$ shear at the blunt boundary of the magnetic island (value is open). Shallow penetration of heat pulse occurs due to the slight turbulence spreading with high $E \times B$ shear at the sharp edge of the magnetic island (valve is closed). The turbulence spreading into 
the magnetic island's O-point from the magnetic island's X-point causes self-regulated oscillation of transport and topology of magnetic islands (Ida et al. 2015b).

\subsection{Turbulence spreading into the scrape-off layer (SOL)}

The scrape-off layer (SOL) is the region where the temperature gradient perpendicular to the magnetic field is smaller than the critical gradient for turbulence excitation (i.e. stable region) because the heat flux parallel to the magnetic field is dominant. Therefore, the turbulence observed in the SOL is mainly turbulence propagated from the pedestal region at the boundary by turbulence spreading. The $E \times B$ flow shear modulation experiment was performed in the Tokamak de la Junta II (TJ-II) using modulated biasing to distinguish the locally driven turbulence and spreading turbulence. As seen in the contour of poloidal phase velocity in Fig. 22a, a strong $E \times B$ flow shear appears at the plasma boundary $\left(r-r_{0}=0\right)$ when negative biasing is
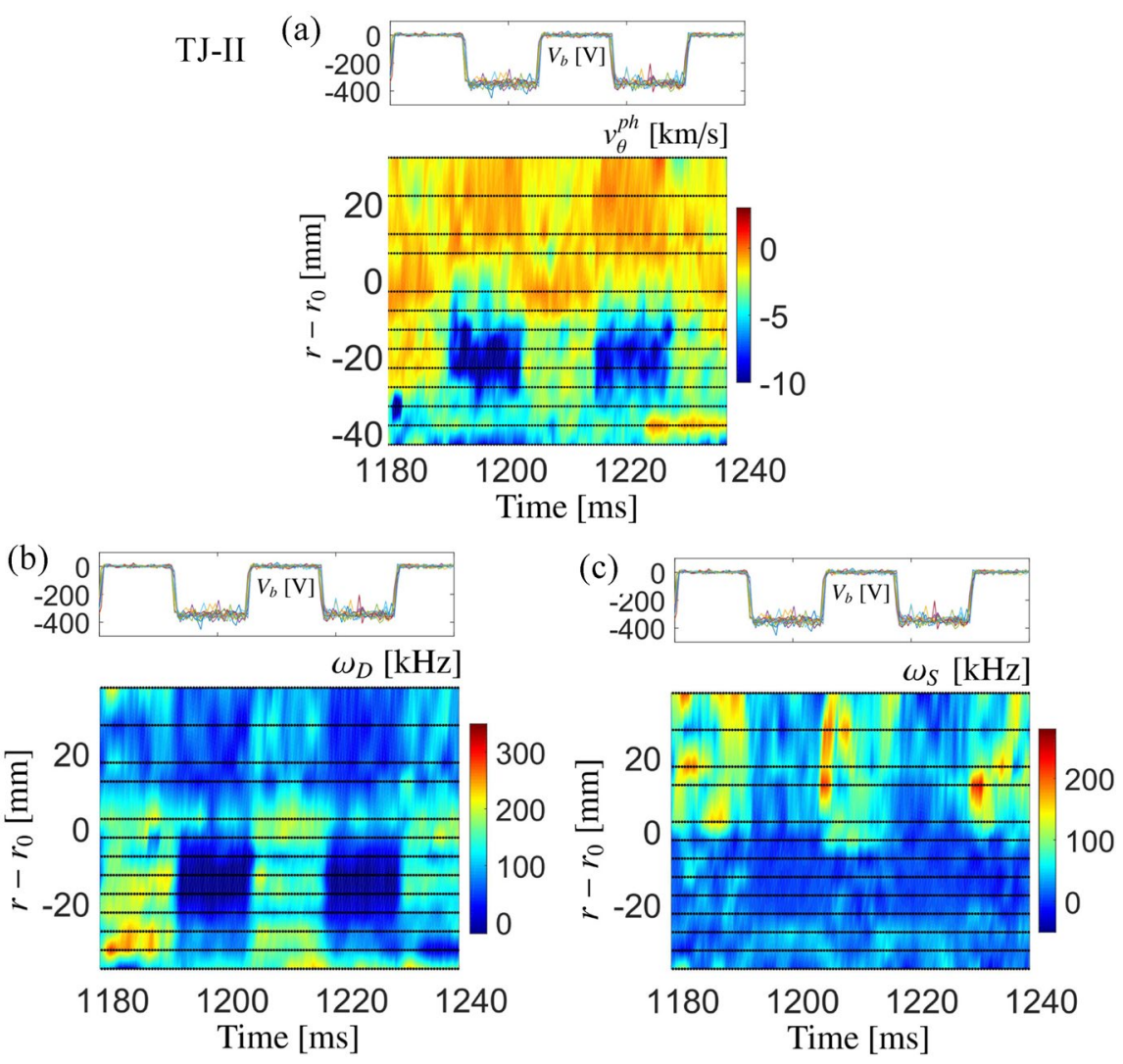

Fig. 22 a Turbulence phase velocity, growth rate of $\mathbf{b}$ local turbulence drive and $\mathbf{c}$ turbulence spreading in plasma boundary region with modulation of edge biasing (from Figs. 6 and 8 in (Grenfell et al. 2019)) 
applied. Here the region for $r-r_{0}>0$ is SOL and the region for $r-r_{0}<0$ is a pedestal. The evolution of turbulent energy is given by

$$
\frac{1}{2} \frac{\partial}{\partial t}\left\langle\tilde{n}^{2}\right\rangle=-\left\langle\frac{\partial n}{\partial r}\right\rangle\left\langle\tilde{v}_{r} \tilde{n}\right\rangle-\frac{1}{2} \frac{\partial}{\partial r}\left\langle\tilde{v}_{r} \tilde{n}^{2}\right\rangle .
$$

The first term RHS of equation (3) is related to the local drive of turbulence by the background (gradient), while the second term is a non-local nonlinear term related to turbulence spreading. Figure 22b, c shows the rate of locally driven turbulence drive, $\omega_{D}$, and the rate of spreading turbulence drive, $\omega_{S}$, defined as

$$
\begin{gathered}
\omega_{D}=-\frac{2\left\langle\frac{\partial n}{\partial r}\right\rangle\left\langle\tilde{v}_{r} \tilde{n}\right\rangle}{\left\langle\tilde{n}^{2}\right\rangle} \\
\omega_{S}=-\frac{\frac{\partial}{\partial r}\left\langle\tilde{v}_{r} \tilde{n}^{2}\right\rangle}{\left\langle\tilde{n}^{2}\right\rangle} .
\end{gathered}
$$

The locally driven turbulence is significantly reduced in the pedestal but only slightly decreases in the SOL when the negative biasing is applied. In contrast, the spreading turbulence is significantly reduced in the SOL but there is no change in the pedestal even for the negative biasing. These results show that the $E \times B$ flow shear at the plasma boundary reduces the locally driven turbulence in the pedestal, blocks the turbulence spreading, and reduces spreading turbulence in the SOL. The block of turbulence spreading by the $E \times B$ flow shear is also observed in the H-mode (Estrada et al. 2011b).

Turbulence spreading into the edge stochastic magnetic layer, induced by magnetic fluctuation has been reported in the LHD (Kobayashi et al. 2021). The turbulence spreading into the SOL region is blocked by the large second derivative of the pressure gradient. When the magnetic fluctuation appears at the boundary, the turbulence spreading is enhanced, and density fluctuation in the SOL region increases. The increase of density fluctuation in this layer results in broadening and reducing the peak divertor heat load. The reduction of the divertor heat load by turbulence spreading at the plasma boundary is a beneficial impact of turbulence spreading in nuclear fusion research.

\section{Summary}

The non-local phenomena and non-local transport are commonly observed in toroidal plasma in the tokamak and helical systems. The cold pulse's transient core temperature rise is widely seen in the low-density plasma (linear ohmic confinement regime in the tokamak and a low collisionality regime in the helical system). This core temperature rise is due to the transient improvement of confinement (reduction of thermal diffusivity) at mid-radius and exhibits hysteresis in the gradient-flux relation. The hysteresis in the gradient-flux relation 
is also commonly observed at mid-radius in the plasma with central modulated $\mathrm{ECH}$. This hysteresis is due to the immediate increase of turbulence level before the temperature gradient increase at the onset of ECH. Therefore the hysteresis appears in the gradient-flux relation, not in the turbulence-flux relation. These hystereses in the gradient-flux relation, observed in the cold pulse experiment and modulation ECH experiment, are clear evidence for non-local transport. The other non-local phenomenon is a simultaneous increase and decrease of the temperature gradient at the core and edge during the formation of the ITB, which is called a see-saw transport.

There are two categories of mechanism of non-local transports. One is the radial propagation of density gradient, temperature gradient, and turbulence. The radial propagation of turbulence is called turbulence spreading. In a case where the turbulence spreading is intermittent, fast, and accompanied by the fast radial propagation of a sharp local temperature gradient above the critical gradient, it is called an avalanche. The amplitude of the avalanche is comparable to the amplitude of a steadystate turbulence level, and avalanche events are buried by turbulence in most of the experiments. The other is the radial coupling of micro-scale turbulence between different locations by the turbulence mediator. The candidates for the turbulence mediator are meso/macro-scale turbulence, MHD oscillations, and zonal flow. The energy transfers between the micro-scale and macro-scale turbulence and between the micro-scale and zonal flow are identified in the experiment.

The non-local transport plays an important role even in a steady state because the turbulence spreading has a substantial impact on the structure formation in the plasma. Turbulence spreading occurs from an unstable region to a stable region in the plasma. For example, the turbulence excited outside the ITB spreads into the ITB region across the so-called ITB foot. Turbulence spreading causes deeper penetration of turbulence into the ITG region and a smooth change in temperature gradient (foot structure becomes obscure). When the turbulence spreading is blocked by the $E \times B$ flow shear at the foot point, the turbulence inside the ITB is further reduced, and the temperature gradient and the $E \times B$ flow shear increases (foot structure becomes prominent). The interplay between turbulence spreading and the $E \times B$ flow shear causes a structure bifurcation of the ITB (curvature bifurcation) The transport bifurcation due to the same process is also observed inside the magnetic island. (low and high accessibility states bifurcation). The block of turbulence spreading by the $E \times B$ flow shear is observed in the region where the magnetic topology changes, such as the boundary of the magnetic island and the last closed flux surface (LCFS). The non-local transport nature, which is revealed by the research in transient phenomena of toroidal plasma, played a critical role in the structure formation in the steady-state phase.

Acknowledgements The author would like to express his sincere thanks to Drs. M. Yoshinuma, T. Kobayashi, N. Tamura, S. Inagaki, Y. Sakamoto, K. Kamiya, M. Yoshida, T.E. Evans, G.R. McKee, M. Ono, with whom the author has worked together in the LHD, JT-60U, and DIII-D and Drs. K. Itoh, P.H. Diamond, C. Hidalgo for their stimulating discussions. This article is dedicated to the memory of Dr. T.E. Evans (General Atomics) who pioneered magnetic island physics.

Funding This work is supported by Grant-in-Aid for Scientific Research (No. 21H04973) of JSPS Japan. 


\section{Declarations}

Conflicts of interest On behalf of all the authors, the corresponding author states that there is no conflict of interest.

Open Access This article is licensed under a Creative Commons Attribution 4.0 International License, which permits use, sharing, adaptation, distribution and reproduction in any medium or format, as long as you give appropriate credit to the original author(s) and the source, provide a link to the Creative Commons licence, and indicate if changes were made. The images or other third party material in this article are included in the article's Creative Commons licence, unless indicated otherwise in a credit line to the material. If material is not included in the article's Creative Commons licence and your intended use is not permitted by statutory regulation or exceeds the permitted use, you will need to obtain permission directly from the copyright holder. To view a copy of this licence, visit http://creativecommons.org/ licenses/by/4.0/.

\section{References}

A. Ashourvan, P.H. Diamond, Phys. Rev. E 94, 051202(R) (2016). https://doi.org/10.1103/PhysRevE.94. 051202

D. del-Castillo-Negrete, B.A. Carreras, V.E. Lynch, Phys. Rev. Lett. 94, 065003 (2005). https://doi.org/ 10.1103/PhysRevLett.94.065003

W. Chen, Y. Xu, X.T. Ding, Z.B. Shi, M. Jiang, W.L. Zhong, X.Q. Ji, Nucl. Fusion 56, 044001 (2016). https://doi.org/10.1088/0029-5515/56/4/044001

M.J. Choi, H. Jhang, J.M. Kwon, J. Chung, M. Woo, L. Qi, S. Ko, T.S. Hahm, H.K. Park, H.S. Kim, J. Kang, J. Lee, M. Kim, G.S. Yun, Nucl. Fusion 59, 086027 (2019). https://doi.org/10.1088/17414326/ab247d

M.J. Choi, L. Bardóczi, J.M. Kwon, T.S. Hahm, H.K. Park, J. Kim, M. Woo, B.H. Park, G.S. Yun, E. Yoon, G. McKee, Nat. Commun. 12, 375 (2021). https://doi.org/10.1038/s41467-020-20652-9

P.H. Diamond, Y.-M. Liang, B.A. Carreras, P.W. Terry, Phys. Rev. Lett. 72, 2565-2568 (1994). https:// doi.org/10.1103/PhysRevLett.72.2565

P.H. Diamond, T.S. Hahm, Phys. Plasmas 2, 3640 (1995). https://doi.org/10.1063/1.871063

P.H. Diamond, A. Hasegawa, K. Mima, Plasma Phys. Control. Fusion 53, 124001 (2011). https://doi.org/ 10.1088/0741-3335/53/12/124001

G. Dif-Pradalier, G. Hornung, P. Ghendrih, Y. Sarazin, F. Clairet, L. Vermare, P.H. Diamond, J. Abiteboul, T. Cartier-Michaud, C. Ehrlacher, D. Estéve, X. Garbet, V. Grandgirard, Ö.D. Gürcan, P. Hennequin, Y. Kosuga, G. Latu, P. Maget, P. Morel, C. Norscini, R. Sabot, A. Storelli, Phys. Rev. Lett. 114, 085004 (2015). https://doi.org/10.1103/PhysRevLett.114.085004

G. Dif-Pradalier, G. Hornung, X. Garbet, P. Ghendrih, V. Grandgirard, G. Latu, Y. Sarazin, Nucl. Fusion 57, 066026 (2017). https://doi.org/10.1088/1741-4326/aa6873

A. Fujisawa, K. Itoh, H. Iguchi, K. Matsuoka, S. Okamura, A. Shimizu, T. Minami, Y. Yoshimura, K. Nagaoka, C. Takahashi, M. Kojima, H. Nakano, S. Ohsima, S. Nishimura, M. Isobe, C. Suzuki, T. Akiyama, K. Ida, K. Toi, Phys. Rev. Lett. 93, 165002 (2004). https://doi.org/10.1103/PhysRevLett. 93.165002

T. Estrada, C. Hidalgo, T. Happel, P.H. Diamond, Phys. Rev. Lett. 107, 245004 (2011). https://doi.org/10. 1103/PhysRevLett.107.245004

T. Estrada, C. Hidalgo, T. Happel, Nucl. Fusion 51, 0032001 (2011). https://doi.org/10.1088/0029-5515/ $51 / 3 / 032001$

A. Fujisawa, Nucl. Fusion 49, 013001 (2009). https://doi.org/10.1088/0029-5515/49/1/013001

K.W. Gentle, W.L. Rowan, R.V. Bravenec, G. Cima, T.P. Crowley, H. Gasquet, G.A. Hallock, J. Heard, A. Ouroua, P.E. Phillips, D.W. Ross, P.M. Schoch, C. Watts, Phys. Rev. Lett. 74, 3620-3623 (1995). https://doi.org/10.1103/PhysRevLett.74.3620

K.W. Gentle, M.E. Austin, J.C. DeBoo, T.C. Luce, C.C. Petty, Phys. Plasmas 13, 012311 (2006). https:// doi.org/10.1063/1.2163251 
G. Grenfell, B.P. van Milligen, U. Losada, W. Ting, B. Liu, C. Silva, M. Spolaore, C. Hidalgo, Nucl. Fusion 59, 016018 (2019). https://doi.org/10.1088/1741-4326/aaf034

W. Guo, P.H. Diamond, D.W. Hughes, L. Wang, A. Ashourvan, Plasma Phys. Control. Fusion 61, 105002 (2019). https://doi.org/10.1088/1361-6587/ab3831

Ö.D. Gürcan, P.H. Diamond, T.S. Hahm, Z.Z. Lin, Phys. Plasmas 12, 032303 (2005). https://doi.org/10. 1063/1.1853385

Ö.D. Gürcan, P.H. Diamond, Phys. Plasmas 13, 052306 (2006). https://doi.org/10.1063/1.2180668

T.S. Hahm, P.H. Diamond, Z. Lin, K. Itoh, S.-I. Itoh, Plasma Phys. Control. Fusion 46, A323-A333 (2004). https://doi.org/10.1088/0741-3335/46/5A/036

T.S. Hahm, P.H. Diamond, J. Kor. Phys. Soc. 73, 747-792 (2018). https://doi.org/10.3938/jkps.73.747

T.S. Hahm, Y.J. Kim, P.H. Diamond, G.J. Choi, Phys. Plasmas 28, 022302 (2021). https://doi.org/10. 1063/5.0036583

F. Hariri, V. Naulin, J. Juul Rasmussen, G.S. Xu, N. Yan, Phys. Rev. Lett. 23, 052512 (2016). https:// doi.org/10.1063/1.4951023

K. Ida, Y. Sakamoto, H. Takenaga, N. Oyama, K. Itoh, M. Yoshinuma, S. Inagaki, T. Kobuchi, A. Isayama, T. Suzuki, T. Fujita, G. Matsunaga, Y. Koide, M. Yoshida, S. Ide, Y. Kamada, Phys. Rev. Lett. 101, 055003 (2008). https://doi.org/10.1103/PhysRevLett.101.055003

K. Ida, Y. Sakamoto, S. Inagaki, H. Takenaga, A. Isayama, G. Matsunaga, R. Sakamoto, K. Tanaka, S. Ide, T. Fujita, H. Funaba, S. Kubo, M. Yoshinuma, T. Shimozuma, Y. Takeiri, K. Ikeda, C. Michael, T. Tokuzawa, Nucl. Fusion 49, 015005 (2009). https://doi.org/10.1088/0029-5515/49/1/ 015005

K. Ida, Y. Sakamoto, S. Inagaki, H. Takenaga, A. Isayama, G. Matsunaga, R. Sakamoto, K. Tanaka, S. Ide, T. Fujita, H. Funaba, S. Kubo, M. Yoshinuma, T. Shimozuma, Y. Takeiri, K. Ikeda, C. Michael, T. Tokuzawa, Nucl. Fusion 49, 095024 (2009). https://doi.org/10.1088/0029-5515/49/9/ 095024

K. Ida, Z. Shi, H.J. Sun, S. Inagaki, K. Kamiya, J.E. Rice, N. Tamura, P.H. Diamond, G. Dif-Pradalier, X.L. Zou, K. Itoh, S. Sugita, O.D. Gürcan, T. Estrada, C. Hidalgo, T.S. Hahm, A. Field, X.T. Ding, Y. Sakamoto, S. Oldenbürger, M. Yoshinuma, T. Kobayashi, M. Jiang, S.H. Hahn, Y.M. Jeon, S.H. Hong, Y. Kosuga, J. Dong, S.-I. Itoh, Nucl. Fusion 55, 013022 (2015). https:// doi.org/10.1088/0029-5515/55/1/013022

K. Ida, T. Kobayashi, T.E. Evans, S. Inagaki, M.E. Austin, M.W. Shafer, S. Ohdachi, Y. Suzuki, S.-I. Itoh, K. Itoh, Sci. Rep. 5, 16165 (2015). https://doi.org/10.1038/srep16165

K. Ida, T. Kobayashi, M. Yoshinuma, Y. Suzuki, Y. Narushima, T.E. Evans, S. Ohdachi, H. Tsuchiya, S. Inagaki, K. Itoh, Nucl. Fusion 56, 092001 (2016). https://doi.org/10.1088/0029-5515/56/9/ 092001

K. Ida, T. Fujita, Plasma Phys. Control. Fusion 60, 033001 (2018). https://doi.org/10.1088/1361$6587 / \mathrm{aa} 9 \mathrm{~b} 03$

K. Ida, T. Kobayashi, M. Ono, T.E. Evans, G.R. McKee, M.E. Austin, Phys. Rev. Lett. 120, 245001 (2018). https://doi.org/10.1103/PhysRevLett.120.245001

K. Ida, Adv. Phys. X 120, 245001 (2020). https://doi.org/10.1080/23746149.2020.1801354

Y. Idomura, H. Urano, N. Aiba, S. Tokuda, Nucl. Fusion 49, 065029 (2009). https://doi.org/10.1088/ 0029-5515/49/6/065029

S. Inagaki, T. Tokuzawa, K. Itoh, K. Ida, S.-I. Itoh, N. Tamura, S. Sakakibara, N. Kasuya, A. Fujisawa, S. Kubo, T. Shimozuma, T. Ido, S. Nishimura, H. Arakawa, T. Kobayashi, K. Tanaka, Y. Nagayama, K. Kawahata, S. Sudo, H. Yamada, A. Komori, Phys. Rev. Lett. 107, 115001 (2011). https://doi.org/10.1103/PhysRevLett.107.115001

S. Inagaki, T. Kobayashi, K. Itoh, T. Tokuzawa, S.-I. Itoh, K. Ida, N. Tamura, N. Kasuya, A. Fujisawa, S. Kubo, T. Shimozuma, M. Yagi, K. Tanaka, Y. Nagayama, K. Kawahata, H. Yamada, J. Phys. Soc. Jpn. 81, 034501 (2012). https://doi.org/10.1143/JPSJ.81.034501

S. Inagaki, N. Tamura, T. Tokuzawa, K. Ida, T. Kobayashi, T. Shimozuma, S. Kubo, H. Tsuchiya, Y. Nagayama, K. Kawahata, S. Sudo, A. Fujisawa, K. Itoh, S.-I. Itoh, Nucl. Fusion 52, 023022 (2012). https://doi.org/10.1088/0029-5515/52/2/023022

S. Inagaki, T. Tokuzawa, N. Tamura, S.-I. Itoh, T. Kobayashi, K. Ida, T. Shimozuma, S. Kubo, K. Tanaka, T. Ido, A. Shimizu, H. Tsuchiya, N. Kasuya, Y. Nagayama, Nucl. Fusion 53, 113006 (2013). https://doi.org/10.1088/0029-5515/53/11/113006

S. Inagaki, T. Tokuzawa, T. Kobayashi, S.-I. Itoh, K. Itoh, K. Ida, A. Fujisawa, S. Kubo, T. Shimozuma, N. Tamura, N. Kasuya, H. Tsuchiya, Y. Nagayama, Nucl. Fusion 54, 114014 (2014). https://doi.org/10.1088/0029-5515/54/11/114014 
S.-I. Itoh, K. Itoh, Sci. Rep. 2, 860 (2012). https://doi.org/10.1038/srep00860

K. Itoh, S. Toda, A. Fujisawa, S.-I. Itoh, M. Yagi, A. Fukuyama, P.H. Diamond, K. Ida, Phys. Plasmas 14, 1020702 (2007). https://doi.org/10.1063/1.2435310

K. Itoh, S.-I. Itoh, M. Yagi, A. Fukuyama, J. Plasma Fusion Res. SERIES 8, 119-121 (2009)

S. Jolliet, Y. Idomura, Nucl. Fusion 52, 023026 (2012). https://doi.org/10.1088/0029-5515/52/2/ 023026

M. Kikuchi, M. Azumi, Rev. Mod. Phys. 84, 1807-1854 (2012). https://doi.org/10.1103/RevMo dPhys.84.1807

E.J. Kim, P.H. Diamond, Phys. Rev. Lett. 90, 185006 (2003). https://doi.org/10.1103/PhysRevLett.90. 185006

F. Kin et. al., Observation of bursty fluctuation associating to avalanche heat transport in JT-60U (22 Ca04) presented at 38th annual meeting of Plasma and Fusion Research 22-25 November 2021

T. Kobayashi, K. Itoh, T. Ido, K. Kamiya, S.-I. Itoh, Y. Miura, Y. Nagashima, A. Fujisawa, S. Inagaki, K. Ida, K. Hoshino, Phys. Rev. Lett. 111, 035002 (2013). https://doi.org/10.1103/PhysRevLett. 111.035002

T. Kobayashi, K. Itoh, T. Ido, K. Kamiya, S.-I. Itoh, Y. Miura, Y. Nagashima, A. Fujisawa, S. Inagaki, K. Ida, N. Kasuya, K. Hoshino, Nucl. Fusion 54, 073017 (2014). https://doi.org/10.1088/00295515/54/7/073017

T. Kobayashi, H. Takahashi, K. Nagaoka, M. Sasaki, M. Yokoyama, R. Seki, M. Yoshinuma, K. Ida, Plasma Phys. Control. Fusion 61, 085005 (2019). https://doi.org/10.1088/1361-6587/ab221c

M. Kobayashi, K. Tanaka, K. Ida, Y. Takemura, Y. Hayashi, T. Kinoshita, submitted to Phys (Rev, Lett, 2021)

M. Leconte, T. Kobayashi, Phys. Plasmas 28, 014503 (2021). https://doi.org/10.1063/5.0030018

Z. Lin, T.S. Hahm, Phys. Plasmas 11, 1099 (2004). https://doi.org/10.1063/1.1647136

P. Mantica, P. Galli, G. Gorini, G.M.D. Hogeweij, J. de Kloe, N.J. Lopes Cardozo, Phys. Rev. Lett. 82, 5048-5051 (1999). https://doi.org/10.1103/PhysRevLett.82.5048

B.P. van Milligen, T. Kalhoff, M.A. Pedrosa, C. Hidalgo, Nucl. Fusion 48, 115003 (2008). https://doi. org/10.1088/0029-5515/48/11/115003

Y. Nagashima, S.-I. Itoh, M. Yagi, K. Itoh, A. Fujisawa, K. Hoshino, K. Shinohara, K. Uehara, Y. Kusama, A. Ejiri, Y. Takase, Rev. Sci. Instrum. 77, 045110 (2006). https://doi.org/10.1063/1. 2194016

P.A. Politzer, M.E. Austin, M. Gilmore, G.R. McKee, T.L. Rhodes, C.X. Yu, E.J. Doyle, T.E. Evans, R.A. Moyere, Phys. Plasmas 9, 1962 (2002). https://doi.org/10.1063/1.1452730

L. Qi, T.S. Jae-Min Kwon, S.Y. Hahm, M.J. Choi, Nucl. Fusion 59, 026013 (2019). https://doi.org/10. 1088/1741-4326/aaf5fd

J.E. Rice, C. Gao, M.L. Reinke, P.H. Diamond, N.T. Howard, H.J. Sun, I. Cziegler, A.E. Hubbard, Y.A. Podpaly, W.L. Rowan, J.L. Terry, M.A. Chilenski, L. Delgado-Aparicio, P.C. Ennever, D. Ernst, M.J. Greenwald, J.W. Hughes, Y. Ma, E.S. Marmar, M. Porkolab, A.E. White, S.M. Wolfe, Nucl. Fusion 53, 033004 (2013). https://doi.org/10.1088/0029-5515/53/3/033004

J.E. Rice, J. Citrin, N.M. Cao, P.H. Diamond, M. Greenwald, B.A. Grierson, Nucl. Fusion 60, 105001 (2020). https://doi.org/10.1088/1741-4326/abac4b

J.E. Rice, N.M. Cao, T. Tala, C. Chrystal, M.J. Greenwald, J.W. Hughes, E.S. Marmar, M.L. Reinke, P. Rodriguez Fernandez, A. Salmi, Nucl. Fusion 61, 026013 (2021). https://doi.org/10.1088/ 1741-4326/abcb26

P. Rodriguez-Fernandez, A.E. White, N.T. Howard, B.A. Grierson, G.M. Staebler, J.E. Rice, X. Yuan, N.M. Cao, A.J. Creely, M.J. Greenwald, A.E. Hubbard, J.W. Hughes, J.H. Irby, F. Sciortino, Phys. Rev. Lett. 120, 075001 (2018). https://doi.org/10.1103/PhysRevLett.120.075001

U. Stroth, L. Giannone, H.-J. Hartfuss, Plasma Phys. Control. Fusion 38, 611-618 (1996). https://doi. org/10.1088/0741-3335/38/4/010

N. Tamura, S. Inagaki, K. Ida, T. Shimozuma, S. Kubo, T. Tokuzawa, K. Tanaka, S.V. Neudatchin, K. Itoh, D. Kalinina, S. Sudo, Y. Nagayama, K. Ohkubo, K. Kawahata, A. Komori, Phys. Plasmas 12, 110705 (2005). https://doi.org/10.1063/1.2131047

N. Tamura, S. Inagaki, K. Tanaka, C. Michael, T. Tokuzawa, T. Shimozuma, S. Kubo, R. Sakamoto, K. Ida, K. Itoh, D. Kalinina, S. Sudo, Y. Nagayama, K. Kawahata, A. Komori, Nucl. Fusion 47, 449-455 (2007). https://doi.org/10.1088/0029-5515/47/5/009 
N. Tamura, K. Ida, S. Inagaki, K. Tanaka, T. Tokuzawa, K. Itoh, T. Shimozuma, S. Kubo, H. Tsuchiya, Y. Nagayama, K. Kawahata, S. Sudo, H. Yamada, Contrib. Plasma Phys. 50, 514-519 (2010). https://doi.org/10.1002/ctpp.200900004

H. Takahashi, K. Nagaoka, S. Murakami, M. Osakabe, H. Nakano, K. Ida, T.I. Tsujimura, S. Kubo, T. Kobayashi, K. Tanaka, R. Seki, Y. Takeiri, M. Yokoyama, S. Maeta, M. Nakata, M. Yoshinuma, I. Yamada, R. Yasuhara, T. Ido, A. Shimizu, H. Tsuchiya, T. Tokuzawa, M. Goto, T. Oishi, S. Morita, C. Suzuki, M. Emoto, K. Tsumori, K. Ikeda, M. Kisaki, T. Shimozuma, Y. Yoshimura, H. Igami, R. Makino, T. Seki, H. Kasahara, K. Saito, S. Kamio, K. Nagasaki, T. Mutoh, O. Kaneko, T. Morisaki, Nucl. Fusion 57, 086029 (2017). https://doi.org/10.1088/1741-4326/aa754b

D.L. Yu, Y.L. Wei, L. Liu, J.Q. Dong, K. Ida, K. Itoh, A.P. Sun, J.Y. Cao, Z.B. Shi, Z.X. Wang, Y. Xiao, B.S. Yuan, H.R. Du, X.X. He, W.J. Chen, Q. Ma, S.-I. Itoh, K.J. Zhao, Y. Zhou, J. Wang, X.Q. Ji, W.L. Zhong, Y.G. Li, J.M. Gao, W. Deng, Y.X. Yi Liu, L.W. Yan, Q.W. Yang, X.T. Ding, X.R. Duan, Y. Liu, Nucl. Fusion 56, 056003 (2016). https://doi.org/10.1088/0029-5515/ $56 / 5 / 056003$

M. Xu, G.R. Tynan, P.H. Diamond, P. Manz, C. Holland, N. Fedorczak, S. Chakraborty Thakur, J.H. Yu, K.J. Zhao, J.Q. Dong, J. Cheng, W.Y. Hong, L.W. Yan, Q.W. Yang, X.M. Song, Y. Huang, L.Z. Cai, W.L. Zhong, Z.B. Shi, X.T. Ding, X.R. Duan, Y. Liu, Phys. Rev. Lett. 108, 245001 (2012). https://doi.org/10.1103/PhysRevLett.108.245001

Y. Xu, D. Carralero, C. Hidalgo, S. Jachmich, P. Manz, E. Martines, B. van Milligen, M.A. Pedrosa, M. Ramisch, I. Shesterikov, C. Silva, M. Spolaore, U. Stroth, N. Vianello, Nucl. Fusion 51, 063020 (2011). https://doi.org/10.1088/0029-5515/51/6/063020

Publisher's Note Springer Nature remains neutral with regard to jurisdictional claims in published maps and institutional affiliations. 